\title{
Splicing kinetics and transcript release from the chromatin compartment limit the rate of Lipid A-induced gene expression
}

\author{
AMY PANDYA-JONES, ${ }^{1,2,4}$ DEV M. BHATT, ${ }^{1,2,4}$ CHIA-HO LIN,${ }^{1}$ ANN-JAY TONG, ${ }^{1,2}$ STEPHEN T. SMALE, ${ }^{1,2,4,5}$ \\ and DOUGLAS L. BLACK ${ }^{1,2,3,4,5}$ \\ ${ }^{1}$ Department of Microbiology, Immunology and Molecular Genetics, University of California, Los Angeles, California 90025, USA \\ ${ }^{2}$ Molecular Biology Institute, University of California, Los Angeles, California 90025, USA \\ ${ }^{3}$ Howard Hughes Medical Institute, University of California, Los Angeles, California 90025, USA
}

\begin{abstract}
The expression of eukaryotic mRNAs is achieved though an intricate series of molecular processes that provide many steps for regulating the production of a final gene product. However, the relationships between individual steps in mRNA biosynthesis and the rates at which they occur are poorly understood. By applying RNA-seq to chromatin-associated and soluble nucleoplasmic fractions of RNA from Lipid A-stimulated macrophages, we examined the timing of exon ligation and transcript release from chromatin relative to the induction of transcription. We find that for a subset of genes in the Lipid $A$ response, the ligation of certain exon pairs is delayed relative to the synthesis of the complete transcript. In contrast, $3^{\prime}$ end cleavage and polyadenylation occur rapidly once transcription extends through the cleavage site. Our data indicate that these transcripts with delayed splicing are not released from the chromatin fraction until all the introns have been excised. These unusual kinetics result in a chromatin-associated pool of completely transcribed and $3^{\prime}$-processed transcripts that are not yet fully spliced. We also find that long introns containing repressed exons that will be excluded from the final mRNA are excised particularly slowly relative to other introns in a transcript. These results indicate that the kinetics of splicing and transcript release contribute to the timing of expression for multiple genes of the inflammatory response.
\end{abstract}

Keywords: gene expression; pre-mRNA splicing; splicing kinetics; mRNA maturation; inflammatory response

\section{INTRODUCTION}

Eukaryotic mRNAs mature via a complex series of molecular reactions prior to their export from the nucleus for translation in the cytoplasm. RNA synthesis commences upon the initiation of transcription by RNA polymerase II (Pol II) from a promoter, followed by elongation through the template DNA, and termination often many kilobases downstream (Selth et al. 2010). Early in transcription, a 7-methyl guanosine cap is added to the $5^{\prime}$ end of precursor messenger RNAs (pre-mRNAs) (Cho et al. 1997; McCracken et al. 1997; Shuman 2001). Most nascent Pol II transcripts also contain introns that must be excised during mRNA maturation (Matlin and Moore 2007; Pandya-Jones and Black 2009; Perales and Bentley 2009; Wahl et al. 2009). It is thought that initial spliceosome assembly on the pre-mRNA occurs

${ }^{4}$ These authors contributed equally to this work.

${ }^{5}$ Corresponding authors

E-mail dougb@microbio.ucla.edu

E-mail smale@mednet.ucla.edu

Article published online ahead of print. Article and publication date are at http://www.rnajournal.org/cgi/doi/10.1261/rna.039081.113. during synthesis, and some introns on long transcripts are removed prior to complete transcription (Osheim et al. 1985; LeMaire and Thummel 1990; Bauren and Wieslander 1994; Tennyson et al. 1995; Wetterberg et al. 1996; Lacadie et al. 2006; Listerman et al. 2006). Once transcription through the gene is complete, the pre-mRNA must be cleaved and polyadenylated to generate its mature $3^{\prime}$ end (Chan et al. 2011). Full-length transcripts are released from the DNA template to allow export to the cytoplasm (Stewart 2010). These multiple reactions provide many potential steps at which to regulate gene expression, and some of these steps are coupled to each other, either kinetically or mechanistically (Hocine et al. 2010; Hsin and Manley 2012). However, the mechanisms regulating individual steps and how they each contribute to the output of the final product are not well understood.

Our incomplete picture of the gene expression pathway is in part due to limited information on the reaction kinetics of many of the steps. The rate of transcription elongation has been estimated using a variety of approaches (Ardehali and Lis 2009). However, endogenous rates of RNA processing reactions in mammalian systems have proven more difficult to measure, and how they affect the time required for a pre- 
mRNA to be matured for export is not known (Grunwald and Singer 2010; Schmid and Jensen 2010; Rabani et al. 2011). The kinetics of excision for individual introns have been described, but larger scale analyses are limited (Kessler et al. 1993; Bauren and Wieslander 1994; Tennyson et al. 1995; Singh and Padgett 2009; Hicks et al. 2010). Nevertheless, understanding the sequence of events in mRNA production will be essential before we can accurately describe their mechanisms of regulation.

We recently reported the application of RNA sequencing (RNA-seq) to understanding the Lipid A-induced transcriptional response in mouse bone marrow-derived macrophages (Bhatt et al. 2012). This analysis of RNA from the chromatin, soluble nucleoplasmic, and cytoplasmic compartments over time after Lipid A stimulation enabled us to characterize the pro-inflammatory gene expression program at a new level of detail. Notably, it was possible to follow the transcription kinetics of individual genes from initiation to completion on chromatin, the release of transcripts into the nucleoplasm, followed by appearance of mRNAs in the cytoplasm. The varying kinetics by which transcripts proceeded through these steps permitted a quantitative and comprehensive categorization of different components of the Lipid A response. An interesting feature of these data was the apparent abundance of fully transcribed and only partially spliced RNAs that remained associated with chromatin, beyond the time required for transcription to complete. This implied that transcript release from this compartment might be a slower step than was previously thought and presented the question of what was necessary for release to occur.

Using this Lipid A induction system, we sought to examine the timing of the splicing reaction and transcript release for genes of the inflammatory response. We find that particular introns in this regulatory cascade are often excised with markedly slower kinetics than has been measured in constitutive transcripts and that incompletely processed RNAs are held within the chromatin fraction until fully spliced. These results have important implications for understanding mechanisms of splicing regulation and of quality control during mRNA biogenesis. Most notably, our data indicate that transcript release from chromatin plays a substantial role in the timing of inflammatory gene expression.

\section{RESULTS}

\section{Assessing splicing of Lipid A-induced transcripts}

In a recent study, we applied RNA-seq to chromatin-associated, nucleoplasmic, and cytoplasmic RNA isolates from macrophages (Bhatt et al. 2012). Using this RNA sampling, we examined gene responses at $15,30,60$, or 120 min postLipid A treatment (Fig. 1A; Materials and Methods). This work defined groups of Lipid A-responsive genes with different induction kinetics that we tracked from transcription initiation and elongation, through the movement of completed messages from the chromatin to the nucleoplasm and into the cytoplasm. We noted that many introns were present within the chromatin compartment at times when transcription through the gene appeared complete. Using these data sets, we wanted to closely examine the timing of intron removal relative to the initiation of transcription, the completion of transcript synthesis, and the release of the message from chromatin. Our goal was to understand how these different steps contribute to the overall timing of inflammatory gene induction.

In previous studies of fractionated cell nuclei, nascent premRNAs co-purified with the salt, detergent, and urea-resistant pellet while the soluble nuclear extract largely contained complete, spliced messages, as well as many components of nuclear speckles (Wuarin and Schibler 1994; Pandya-Jones and Black 2009; Khodor et al. 2011; Bhatt et al. 2012). The nascent RNAs are released from the pellet by DNase treatment, indicating their association requires intact chromatin (A Pandya-Jones and DL Black, unpubl.). In primary mouse macrophages we found a similar distribution of steady-state RNA populations. For constitutive genes, whose expression was unaffected by Lipid A treatment, nucleoplasmic reads were highly enriched for exonic regions, as would be expected of fully spliced RNA (Supplemental Fig. S1A,B, red). For most of these genes, intron reads in the nucleoplasm were at the level of background contamination from the chromatin, which was estimated from the amount of the known chromatin-associated RNA Xist found in the nucleoplasm (see Materials and Methods; Supplemental Fig. S1C, red). However, this pattern was variable and certain constitutive genes produced higher numbers of nucleoplasmic reads mapping to intronic sequences (Supplemental Fig. S1D, red). It is not clear whether introns in this group of constitutive transcripts are spliced away from the chromatin or are more stable after their excision.

In the chromatin fraction patterns of read accumulation on constitutive genes were more variable. For some genes at steady state, decreasing gradients of read density were observed across the locus, presumably derived from the $5^{\prime}$-to$3^{\prime}$ polarity of transcription, but this was not a general pattern (Supplemental Fig. S1A, green; Bhatt et al. 2012). We also observed some constitutive genes exhibiting higher levels of reads mapping to exons than to adjacent introns (Supplemental Fig. S1B, green), and some genes exhibited almost entirely exon reads even on chromatin (Supplemental Fig. S1E, green). If introns are excised soon after their $3^{\prime}$ exon is synthesized, then exons should be enriched relative to their neighboring intron sequences, particularly toward the $5^{\prime}$ end of the gene. However, again this was not a consistent pattern and many genes exhibited reads that were evenly distributed across the locus regardless of exon or intron location (Supplemental Fig. S1A,B, green and data not shown; see also Ameur et al. 2011; Khodor et al. 2011, 2012). Given the variability in the steady-state read accumulation patterns of constitutive genes, it is difficult to draw conclusions about their rates of RNA synthesis and processing. Thus, we used 

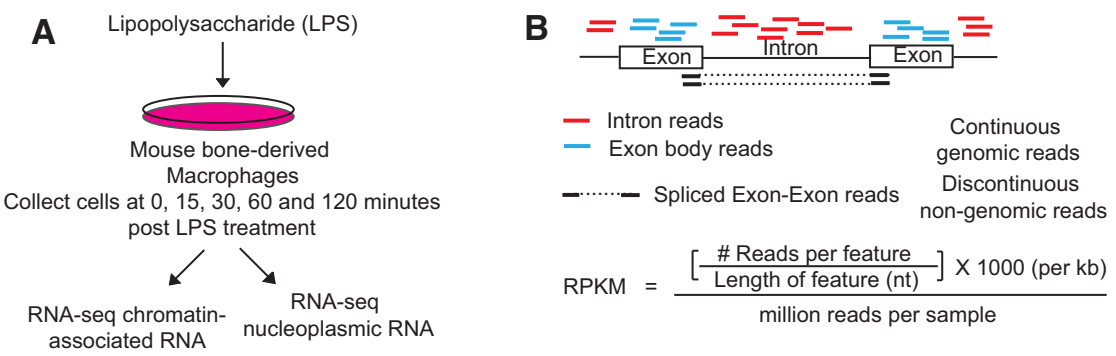

Group A 1 transcripts
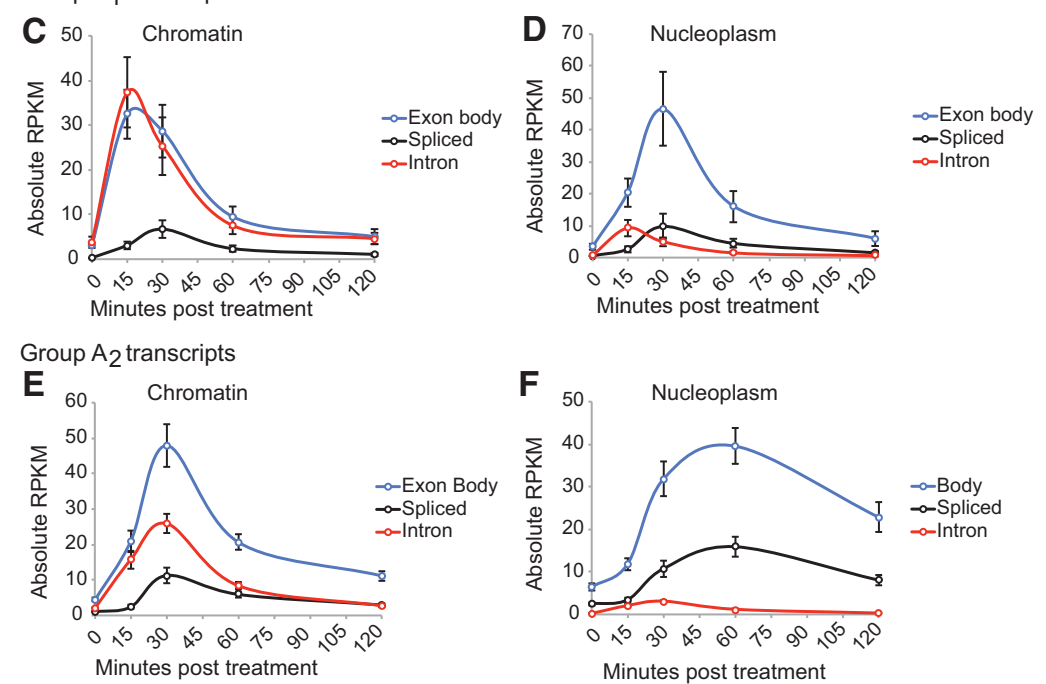

Group B transcripts
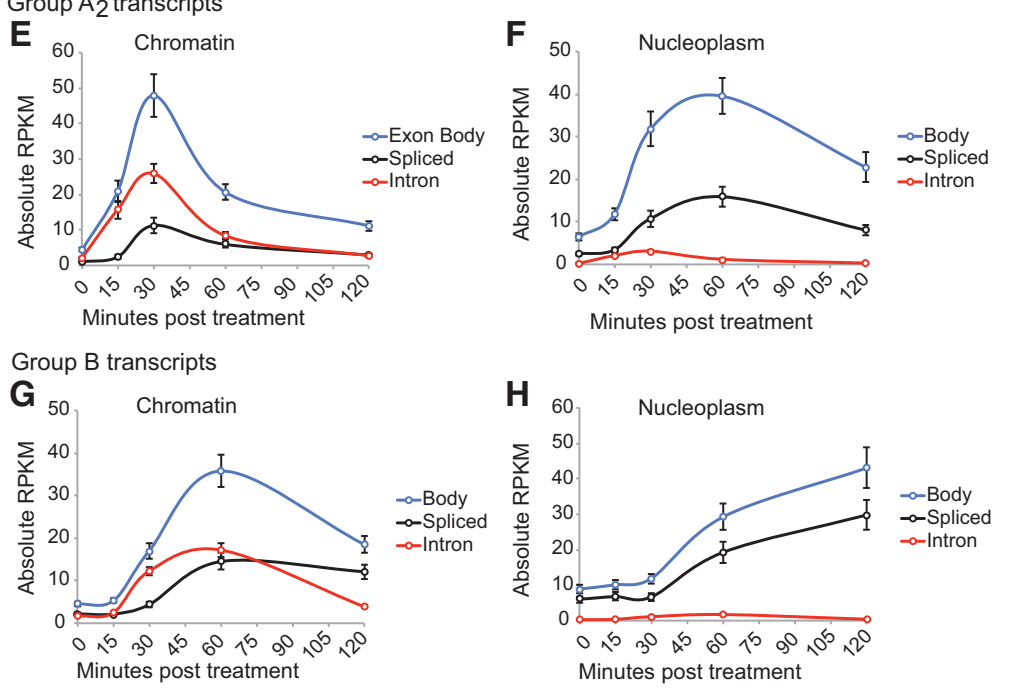

FIGURE 1. RNA synthesis and splicing can be resolved over time on Lipid A-responsive genes. (A) In vitro differentiated mouse macrophages were treated with Lipid A and collected at $0,15,30$, 60 , or 120 min post-simulation. Chromatin-associated and nucleoplasmic RNA fractions from the same biological sample, for each time point, were analyzed by RNA-seq. (B) Reads mapping to genic regions were classified by their location. Continuously mapping genomic reads include the following: exon body reads (blue) that map entirely within exons; intron reads (red) that map entirely within introns; spliced reads (black) mapping discontinuously to the genome represent reads that cross a spliced exon-exon junction. $(C)$ Line graph representing the total RPKM of exon body (blue), intron (red), and spliced reads (black) averaged from all chromatin-associated Group $\mathrm{A}_{1}$ transcripts at each time point post-Lipid A treatment. (D) Same as $C$ except that the exon, intron, and spliced RPKMs were calculated for all nucleoplasmic Group $A_{1}$ transcripts. (E) Same as $C$ except that RPKMs were calculated for all chromatin-associated Group $\mathrm{A}_{2}$ transcripts. (F) Same as D except that RPKMs were calculated for all nucleoplasmic Group $\mathrm{A}_{2}$ transcripts. $(G)$ Same as $C$ except that RPKMs were calculated for all chromatin-associated Group B transcripts. (H) Same as D except that RPKMs were calculated for all nucleoplasmic Group B transcripts. In all figures, error bars represent the standard error of the mean.

the Lipid A-induced genes to examine the rates of intron excision relative to initiation of transcription and release of the completed transcripts from the template.

To identify genes activated by Lipid A treatment, an averaged Reads Per Kilobase of gene per Million mapped reads
(RPKM) value was assigned to each gene, at each time point, for all isoforms within the RefSeq database. This included all exon body, intron body, intron-exon junction, and spliced exonexon reads (Fig. 1B and below). The fold induction was calculated by comparing the isoform with the highest gene RPKM value over the time course relative to the zero time point. We limited our analysis to those genes up-regulated fivefold or more. This included a majority of the previously identified Lipid A-responsive genes, but excluded genes with substantial constitutive activity that show a small increase in expression upon stimulation (RamirezCarrozzi et al. 2009).

Exons constitute only a small portion of the precursor mRNA sequence. To ensure that a majority of exons within the up-regulated transcripts had sufficient coverage for splicing analysis, only genes with an RPKM of $\geq 60$ at one or more time points were selected. At this RPKM threshold, the fraction of exons exhibiting coverage across all nucleotides approached $100 \%$ at peak transcription (data not shown). To assess splicing relative to transcription, we determined RPKM values for four features, at each time point in each fraction (Fig. 1B). The exon body RPKM included only those reads that map entirely within an exon. Since a majority of exons within a precursor are included in the mature message, the exon body RPKM was used as a measure of overall RNA volume in subsequent analyses. The intronic RPKM was calculated from reads that mapped entirely within an intron. We measured splicing by quantifying the reads that map discontinuously with the genome and cross an annotated exon-exon junction (Fig. 1). Note that the transcripts studied here are minimally expressed in macrophages prior to Lipid A exposure, allowing the accumulation of spliced exon reads to be measured over time. This is in contrast to the measurement of steady-state RNA, where ratios of spliced to unspliced introns are inferred from the ratio of reads mapping across a $3^{\prime}$ intron-exon junction to reads within the adjacent exon (Khodor et al. 2011, 2012). Since individual sequences along a transcript generate reads with 
varying efficiencies, we found that this ratio is highly variable when applied to individual introns. By examining genes induced by Lipid A, we could directly measure the rates of splicing from the accumulation of individual exon-exon junction reads over time, and avoid comparing read levels between different short segments of a transcript. We compared the increase in spliced RNA-seq reads with the gain and loss of intron reads over the same time course. Levels of selected exon, intron and spliced exon-exon sequences were also measured by qPCR and RT/PCR and found to match the temporal profiles determined by RNA-seq (data not shown). We also determined RPKM values for reads spanning $3^{\prime}$ intronexon boundaries and found that these reads show similar temporal profiles to those mapping within the intron body (Supplemental Fig. S2).

\section{Splicing of some Lipid A-induced transcripts is delayed relative to transcription}

Genes that made both cutoffs (equal to or greater than fivefold induction and RPKM $\geq 60$ ) were grouped by their kinetics of induction, as described previously (Fig. 1C-H; Bhatt et al. 2012). The 11 Group $A_{1}$ genes reached peak RNA volume on the chromatin within 15 min (Figs. 1C, blue; 2A, green; Supplemental Fig. S3A,E, green). This group included the immediate early genes, such as $c$-Fos and Nfkbid. Group $\mathrm{A}_{2}$ included 34 genes whose RNA accumulation on chromatin peaked at 30 $\min$ (Figs. 1E, blue; $3 \mathrm{~A}$, green). An additional 23 genes that reached maximal transcriptional volume at $60 \mathrm{~min}$ were classed as Group B genes (Figs. 1G, blue; 4A; Supplemental Fig. S4A, green). We focused our splicing analyses on these three gene sets because each of them exhibits a burst of activity upon Lipid A stimulation and returns to near basal transcription within the 2-h time course, thereby allowing us to track the processing and movement of transcripts within the nuclear fractions. Additional classes of Lipid A-responsive genes (Groups C-F) did not return to basal transcription levels during the time course, precluding further splicing analysis (data not shown). Characterization of the expression kinetics of genes comprising Groups $A_{1}-F$ is presented elsewhere (Bhatt et al. 2012).

To determine the timing of splicing relative to transcription initiation, the exon body RPKM was compared with the intron and the spliced exon RPKMs over time. We find that Group $\mathrm{A}_{1}$ genes show similar patterns of exon body and intron body read accumulation on the chromatin between 0 and $15 \mathrm{~min}$ (Fig. 1C, blue and red). Transcriptional volume on these genes is maximal at $15 \mathrm{~min}$, when the majority of $A_{1}$ transcripts appear to be completely synthesized (Fig. 2A; Supplemental Fig. S3A,E, green; and data not shown). Interestingly, the peak of spliced RNA from these genes is not reached until $30 \mathrm{~min}$ (Fig. 1C, cf. the black and blue lines). Thus, chromatin-associated Group $\mathrm{A}_{1}$ transcripts exhibit an observable delay between the accumulation of nascent transcripts and that of the spliced RNA (Fig. 1C, cf. the red and black lines). The length of this delay cannot be precisely measured given rapid induction of $A_{1}$ genes and the time resolution of the experiment. However, the time of maximum increase in spliced reads is offset from the rapid rise in intron and exon reads by 15 min and occurs after transcription through the genes is complete (see below). We find that the nucleoplasmic accumulation of $A_{1}$ transcripts also begins between 0 and $15 \mathrm{~min}$, but does not peak until 30 min (Fig. 1D, blue). The early accumulating nucleoplasmic RNA includes greater numbers of intron body reads than are seen in more slowly induced genes (cf. Fig. 1D and Fig. 1F,
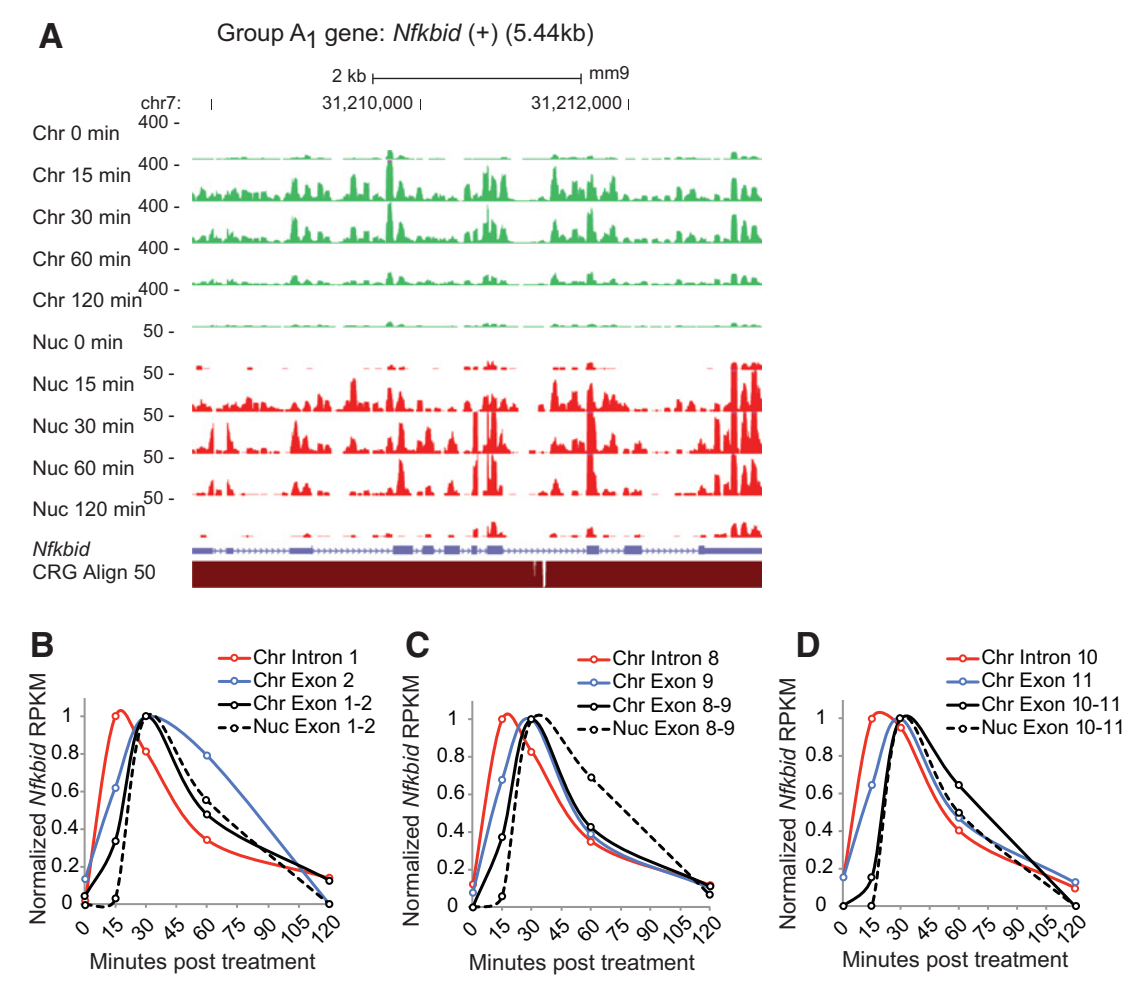

FIGURE 2. Splicing and release of Nfkbid transcripts. (A) Plot of all reads mapping to the $5.44-\mathrm{kb}$ $\mathrm{Nfkbid}$ gene from the chromatin (green) and nucleoplasmic (red) fractions at each time point post-Lipid A treatment aligned to the UCSC genome browser track. The gene structure and mappability for the 50-nt reads are depicted below. $(B-D)$ Line graphs representing the RPKM (normalized to the maximum value) of individual intron (red) or exon (blue) regions within the Nfkbid gene in the chromatin fraction at each time point post-Lipid A treatment. The normalized read numbers mapping to the corresponding exon-exon junctions in the chromatin fraction (black, solid) and nucleoplasm (black, dashed) are also plotted. 
$\mathrm{H}$, red). These reads may derive from excised introns released from the chromatin and/or a minor subset of incompletely spliced transcripts being released into the nucleoplasm. Taken together, these data show that, for Group $A_{1}$ transcripts, the nucleoplasmic accumulation of RNA is delayed relative to its production on the chromatin, but that, since the maximal accumulation of spliced RNA coincides with that of the exon sequences in the nucleoplasm (Fig. 1D, blue and black), the RNA released from the chromatin after $15 \mathrm{~min}$ is largely spliced.

A delay in splicing relative to the onset of transcription is also observed for $\mathrm{A}_{2}$ genes. Production of RNA on the chromatin from $\mathrm{A}_{2}$ genes begins between 0 and 15 min after Lipid A treatment (Fig. 1E, blue) and is accompanied by a commensurate rise in intron reads (Fig. 1E, red). There is no observable accumulation of spliced reads during this time period (Fig. 1E, black). In the next interval (15-30 min), a rapid increase in spliced exon reads is observed (Fig. 1E, black). Intron reads continue to increase during this interval, but at a slower rate than exon body reads, presumably due to splicing. In contrast to the $A_{1}$ transcripts, exon body, intron, and spliced exon reads mapping to $\mathrm{A}_{2}$ genes all peak simultaneously on the chromatin, in spite of the delayed onset of the spliced reads (Fig. 1E). For this group, the nucleoplasmic intron reads are at the level of background contamination from the chromatin (Fig. 1F, red). Notably, the exon body and spliced reads do not reach maximal values in the nucleoplasm until 60 min. Thus, Group $A_{2}$ spliced RNAs exhibit an observable delay in nucleoplasmic accumulation relative to their apparent completion on the chromatin (Fig. 1E,F, black).

Group B transcripts show a pattern of read accumulation on the chromatin similar to Group $\mathrm{A}_{2}$ transcripts, except that the maximal values are shifted to a later time. Exon body and intron reads accumulate at equal rates between 15 and $30 \mathrm{~min}$ (Fig. 1G, blue and red), but substantial spliced read accumulation does not begin until after $30 \mathrm{~min}$ (Fig. $1 G$, black). After this time, accumulation of exon body reads continues, but intron reads begin to plateau, presumably due to splicing. Similar to Group $\mathrm{A}_{2}$, the peak of spliced reads for Group B genes coincides with the peak of total transcript, even though its initial onset is delayed (Fig. 1E,G, cf. black and blue). In the nucleoplasm, both exon body and spliced reads continue to accumulate at $2 \mathrm{~h}$ post-Lipid A stimulation, a time when their abundance on chromatin is decreasing. The intron body reads in the nucleoplasm are at background levels, indicating that these transcripts are nearly completely spliced upon release or are rapidly degraded if released when splicing is incomplete (Fig. $1 \mathrm{H}$, red).

Taken together, these data indicate that transcription, splicing, and chromatin release can be followed over time. Moreover, the three groups of genes differ in their accumulation rates for the different types of reads, but splicing generally precedes nucleoplasmic accumulation. Excision of constitutive introns has been measured as ranging from 2 to $10 \mathrm{~min}$ after synthesis of the $3^{\prime}$ exon in mammalian cells, and faster in yeast (Singh and Padgett 2009; Alexander et al. 2010a; Schmidt et al. 2011). The delayed accumulation of spliced exon reads relative to RNA synthesis in the Lipid Ainduced genes indicates that some introns in this set might exhibit significantly slower than typical excision rates. To measure these rates, we examined individual genes and introns within each of the three groups.

\section{Individual Group $A_{1}$ transcripts exhibit differences in their kinetics of splicing and chromatin release}

Almost all Group $\mathrm{A}_{1}$ genes are $<10 \mathrm{~kb}$ long and contain three or fewer introns (Supplemental Table S1; Bhatt et al. 2012). As expected, this makes the kinetics of their induction, splicing, and release too fast to completely resolve using 15 -min time intervals. For example, at $15 \mathrm{~min}$ the $c$-Fos gene has already reached peak values for intron, exon body, and spliced reads on the chromatin, as well as exon and spliced reads in the nucleoplasm (Supplemental Fig. S3A-D). Interestingly, not all Group $A_{1}$ genes had the same kinetics for splicing and nucleoplasmic accumulation. The gene Nfkbid is only $5.44 \mathrm{~kb}$ long and shows complete transcription across the locus by 15 min (Fig. 2A, green). Nfkbid differs from other Group $A_{1}$ genes in that it contains 10 introns (Fig. 2A, short isoform). We examined the accumulation of reads for several introns and exons along its length. As expected, we found that reads for introns 1,8 , and 10 peak between 15 and 30 min on the chromatin, after which RPKM decreases for all introns (Fig. 2B-D, red). The drop in intronic RPKM after 15 min coincides with a rapid increase in spliced exon reads, which reach their maxima $\sim 30 \mathrm{~min}$ (Fig. 2B-D, black). Reads for the body of exons 2, 9, and 11 show similar rapid increases in the first $15 \mathrm{~min}$ but peak later as these reads derive from both spliced and unspliced RNA (Fig. 2B-D, blue). Thus, even though this gene undergoes rapid transcription to synthesize all of its introns and exons by 15 min, the accumulation of spliced RNA is delayed (Fig. 2BD, black; see also Supplemental Fig. S2 for examples of absolute RPKMs per feature). For these introns, the gain in spliced exons coincides with the decrease in intron sequences, indicating that the excised lariats are short lived. This is seen for many but not all introns (see below and data not shown). Differences in the splicing kinetics of different introns cannot be resolved on this short gene (see below). Interestingly, nucleoplasmic RNA shows no accumulation prior to $15 \mathrm{~min}$ and then rapidly rises to peak sometime after $30 \mathrm{~min}$ (Fig. $2 \mathrm{~B}-\mathrm{D}$, dashed). These data show that both splicing and transcript release occur more slowly than synthesis of full Nfkbid transcripts on the locus. A similar delay in the splicing relative to the accumulation of total reads on chromatin was observed for the longest gene within this group, Atf3 (Supplemental Fig. S3E-G). This confirms our previous observations of full-length but incompletely processed RNAs associated with chromatin whose transcription is induced by Lipid A (Bhatt et al. 2012). We suggest that the delayed nucleoplasmic 
accumulation allows splicing of all introns to be completed prior to release from the chromatin compartment (see below). It is notable that Nfkbid and $c$-Fos are of comparable length and appear to be transcribed at similar rates. However, their rates of splicing differ, and by influencing the time that a transcript is retained on the chromatin these splicing kinetics are determining the rate at which the encoded protein can be expressed in the cytoplasm (Fig. 2; Supplemental Fig. S3).

\section{Nucleoplasmic RNA accumulation can be delayed relative to synthesis and splicing on chromatin}

Although Group $\mathrm{A}_{1}$ genes are all short, the other Lipid A-induced gene groups showed broad length distributions (Supplemental Table S1; Bhatt et al. 2012). We found that transcriptional gradients could be detected on Lipid A-responsive genes that were $>20 \mathrm{~kb}$. As shown for the Group $\mathrm{A}_{2}$ gene Ifrd1, at $15 \mathrm{~min}$ the chromatin-associated reads are more abundant in the $5^{\prime}$ portion of the transcription unit than toward the $3^{\prime}$ end (Fig. 3A, green). By 30 min, reads are more evenly distributed along the length of the gene. Plotting the read accumulation for individual introns, we found that introns 1 and 3 at the $5^{\prime}$ end of the gene (Fig. 3B, blue) accumulate prior to those in the middle (red) or $3^{\prime}$ portions of the transcription unit (black). Intron 2 also showed early accumulation similar to introns 1 and 3 (Fig. 3C, red). The time resolution of the experiment is not sufficient to distinguish more closely spaced introns. Similarly, for the assayable exons the exon body reads and spliced read accumulation could not be resolved from the intron accumulation (Fig. 3B,D,E). These introns are all spliced relatively quickly, although there was a notable delay in the accumulation of spliced exon 2-exon 3 reads after the synthesis of intron 2 (Fig. 3C, black and red). This difference could be due to polymerase pausing, although the profile of RNA accumulation across this region does not show an obvious upstream enrichment that might indicate such a pause.

Interestingly, the Ifrd 1 transcripts display a further delay between the accumulation of spliced reads on the chromatin and their appearance in the nucleoplasm, where they peak at $60 \mathrm{~min}$ (Fig. 3C-E, cf. the solid and dashed black lines). This is in contrast to the transcripts analyzed in the Group $A_{1}$ that are more rapidly released into the nucleoplasm after being spliced. The delay in nucleoplasmic accumulation might reflect the time required for splicing introns in Ifrd1 whose excision is slower than those we were able to assay. Whatever mechanisms are mediating this retention, the lag in Ifrd 1 transcript release from the chromatin is determining when Ifrd1 protein can finally be expressed after stimulation. Even though lfrd 1 transcription is rapidly induced upon stimulation, a substantial delay occurs before its final mRNA is available for translation. Thus, the timing of transcript release will be an important component of the overall regulatory program of inflammation.

\section{Splicing catalysis can occur substantially later than transcript completion}

We next examined long genes that provide greater resolution of splicing and transcription over the time course. Fchsd 2 is $175 \mathrm{~kb}$ long and contains 21 exons (Fig. 4A). Introns within this gene accumulate in sequence, $5^{\prime}$ to $3^{\prime}$, in the chromatin 


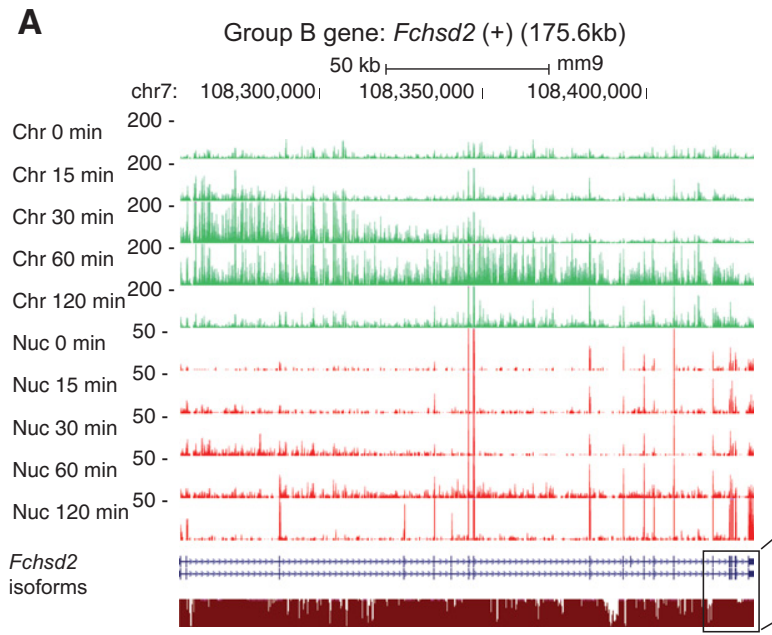

B
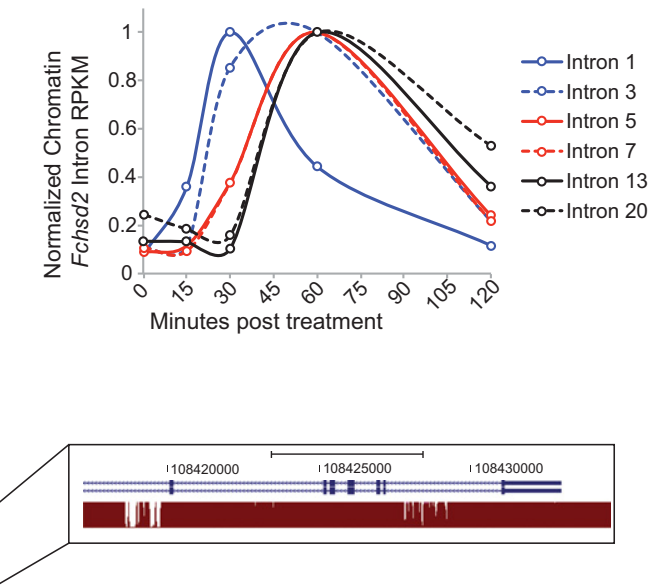
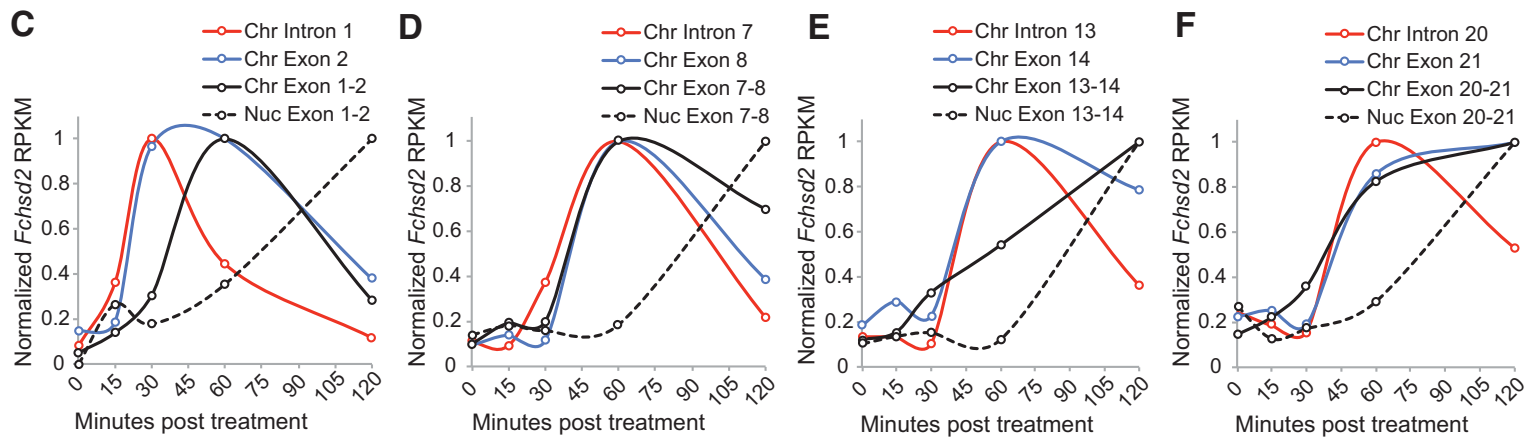

FIGURE 4. Splicing catalysis on long pre-mRNAs can be greatly delayed relative to transcription. (A) Plot of all reads mapping to the $175.2-\mathrm{kb}$ Fchsd2 gene from the chromatin (green) and nucleoplasmic (red) fractions at each time point post-Lipid A treatment aligned to the UCSC genome browser track. The gene structure and mappability for the 50-nt reads are depicted below. For clarity, an enlargement of the $3^{\prime}$ region of the gene is included. (B) Line graph showing the normalized RPKM of introns within chromatin-associated $F c h s d 2$ transcripts. $(C-F)$ Line graphs representing the RPKM of individual intron (red) or exon (blue) regions within the Fchsd2 gene in the chromatin fraction. The reads mapping to the corresponding exon-exon junctions in the chromatin fraction (black, solid) and nucleoplasm (black, dashed) are also shown.

fraction (Fig. 4B). We find that intron 1 and its neighboring exon 2 appear almost simultaneously between 15 and 30 min (Fig. 4C, red and blue). However, there is a significant delay in the accumulation of exon 1-2 spliced reads, which begin to accumulate between 30 and $60 \mathrm{~min}$, coincident with the loss of intron 1 reads (Fig. 4C, cf. red and solid black). Similar results are seen for $5^{\prime}$ proximal intron/exon pairs in $N f k b 1$ and other long genes (Supplemental Fig. S4 and data not shown). A delay in spliced read accumulation could be due, in part, to pausing of the polymerase within an intron and before its downstream exon. However, we do not observe patterns of read enrichment in introns that might be indicative of transcriptional pauses (Core et al. 2008). In addition, published Chromatin Immunoprecipitation (ChIP-seq) data for RNA Pol II in LPS-stimulated macrophages does not indicate polymerase pausing in these regions (De Santa et al. 2009; Escoubet-Lozach et al. 2011). Most significantly, we find that the spliced exon junction reads from $5^{\prime}$ proximal exons display kinetics of accumulation similar to intron and exon reads mapping to the $3^{\prime}$ terminus of the gene (cf. Fig. $4 \mathrm{C}$, black line, and Fig. 4D-F, blue lines, and Supplemental Fig. S4). These results indicate that splicing catalysis for many of these introns coincides roughly with transcript completion (Fig. 4D,E; Bhatt et al. 2012). Once splicing has begun, spliced reads for exons from the $5^{\prime}$ region generally reach maximal abundance earlier than reads for more $3^{\prime}$ spliced exons (Fig. 4C-F; Supplemental Fig. S4). This may indicate that, although splicing catalysis for some introns is delayed relative to intron synthesis, spliceosome assembly is likely initiating during transcription and affecting the relative timing of some splicing events. However, other factors are also clearly affecting the timing of catalysis. The peak RNA volume on the Fchsd2 locus occurs at $60 \mathrm{~min}$, but the exons flanking introns 13 and 20 do not reach maximum ligation until at least $120 \mathrm{~min}$. A similar delay is seen in the splicing of the last exon of $N f k b 1$ (Supplemental Fig. S4). These data indicate that the timing of inflammatory gene product expression, including $N f k b 1$ itself, is not determined solely by the kinetics of transcriptional induction and elongation, but also by the rate of splicing and release of the mRNAs. Importantly, even where splicing 
catalysis is completed quite late, the nucleoplasmic accumulation of RNA coincides with the excision of these slowest introns (Fig. 4C-F; Supplemental Fig. S4).

The changes in read accumulation for different portions of a gene over time can be used to estimate the rate of transcription elongation on Lipid A-induced genes. Examining the Fchsd2 gene, reads at the $5^{\prime}$ end of the gene become detectable at $15 \mathrm{~min}$, whereas those at the $3^{\prime}$ end do not appear until 60 min (Fig. 4A). Thus, RNA Pol II takes at most 45 min to transcribe the 175.2-kb Fchsd2 gene, yielding a minimum elongation rate of $3.89 \mathrm{~kb} / \mathrm{min}$. This value agrees reasonably well with rates of transcription on endogenous mammalian genes determined by a variety of methods (Darzacq et al. 2007; Ardehali and Lis 2009; Singh and Padgett 2009; Brody et al. 2011).

\section{$3^{\prime}$ end cleavage and polyadenylation can precede the splicing of nascent transcripts}

We previously noted that the reads accumulating on chromatin were largely depleted of sequences downstream from the poly(A) site, and much of the RNA appeared to be polyadenylated (Bhatt et al. 2012). To quantify this $3^{\prime}$ processing and assess its timing, we again stimulated primary macrophages with Lipid A and isolated chromatin-associated RNA at 0, 5, $15,20,30$, and $60 \mathrm{~min}$. We then primed reverse transcriptase with primers located either downstream from the poly(A) site (Fig. 5A, blue primer) or at this site after cleavage and polyadenylation (Fig. 5A, red primer). The cDNA was subjected to

A
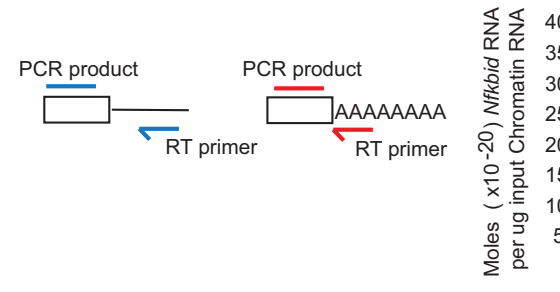

B

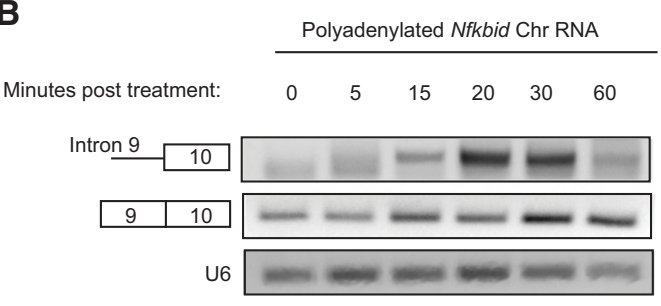

FIGURE 5. Polyadenylation can precede splicing on chromatin. (A) Line graph showing the molar amount of Nfkbid 3' terminal exon per microgram of input chromatin-associated RNA at times post-Lipid A simulation. The RNA was primed for reverse transcription using either a primer downstream from the cleavage site (blue) or one complementary to the poly(A) tail (red). Error bars represent the standard error of the mean. $(B)$ Ethidium bromide stained agarose gel showing the PCR product of the Nfkbid intron 9-exon 10 junction ( 35 cycles) and the corresponding exon 9-exon 10 spliced product ( 35 cycles) within the chromatin fraction at times post-Lipid A stimulation. We note that the intron 9-exon 10 product is short, and appears at the 30-min time point. The band visible at the 0 - and 15-min time points is a primer dimer. A PCR product ( 25 cycles) amplified from the U6 snRNA cDNA served as a loading control.
qPCR to determine the molar amount of $3^{\prime}$ terminal exon sequence in each sample. We analyzed short transcripts synthesized from Group $A_{1}$ and $A_{2}$ genes because their cleavage and polyadenylation status could be assayed early in the time course. Absolute quantification revealed accumulation of both uncleaved and polyadenylated Nfkbid RNA on chromatin over the time course (Fig. 5). We find that polyadenylated transcripts account for $>75 \%$ of the total RNA on chromatin at peak levels of expression, confirming our previous observation of read enrichment upstream of the cleavage and polyadenylation site of most genes (Fig. 5A; Bhatt et al. 2012; and data not shown). This finding is consistent with long-standing observations that polyadenylation occurs rapidly after transcription, and with more recent data that polyadenylated RNAs are retained at a locus after completion of transcription (Derman and Darnell 1974; Nevins and Darnell 1978; Salditt-Georgieff et al. 1980; Brody et al. 2011; Daguenet et al. 2012). Notably, the peak of uncleaved Nfkbid RNA on chromatin occurs at 15 min post-Lipid A stimulation, coincident with the first appearance of full-length transcripts (Fig. 5A). Even at this early time, most of the RNA is already cleaved and polyadenylated. $3^{\prime}$ processed RNA continues to accumulate and peaks $\sim 30$ min post-stimulation, with similar kinetics to the exon body and spliced exon reads (Figs. 2B-D, $5 \mathrm{~A})$. We find that $60 \%$ of all reads mapping to spliced exonexon junctions within Nfkbid accumulate on chromatin between 15 and 30 min post-stimulation (Fig. 2B-D, black). During this time period, $\sim 85 \%$ of the Nfkbid transcripts on chromatin are already polyadenylated (Fig. 5A, red). Thus, a large fraction of intron excision events occur on RNAs that have already been $3^{\prime}$-cleaved and polyadenylated. In the nucleoplasm, uncleaved transcripts are at the level of background, confirming that $3^{\prime}$ end cleavage occurs in association with chromatin (data not shown).

To further confirm that polyadenylated RNAs on chromatin contain introns, we measured the presence of unspliced introns in the polyadenylated Nfkbid RNA samples by PCR. The intron 9-exon 10 splice site was readily detected in this RNA and accumulated with the same kinetics as the polyadenylated $3^{\prime}$ exon, peaking on chromatin at 20-30 min post-stimulation (Fig. 5B). Additional introns in Nfkbid and other Group $A_{1}$ and $A_{2}$ transcripts were also seen in the polyadenylated RNA (data not shown). Thus, splicing catalysis for some introns follows $3^{\prime}$ end maturation. This is consistent with long-standing in vivo labeling data, as well as in vitro data showing that cleavage and $3^{\prime}$ end formation can precede splicing, even though 
splicing is required for release of transcripts from the polymerase (Derman and Darnell 1974; Nevins and Darnell 1978; Bird et al. 2005; Rigo and Martinson 2009; Darnell 2013).

Evidence for polymerase pausing in the final exon of genes has been observed in yeast (Alexander et al. 2010b; Carrillo Oesterreich et al. 2010). However, transcriptional pauses are likely to occur on a time scale too rapid to be assayed in our data.

\section{Introns containing skipped alternative exons are spliced more slowly than adjacent constitutive introns}

We and others previously observed that introns containing skipped exons are present on chromatin at higher levels than other introns, indicating that regions subject to splicing regulation may exhibit slower rates of splicing (PandyaJones and Black 2009; Khodor et al. 2011). Our data here suggest that the intron with the slowest excision rate might determine the release kinetics of its parent transcript. To examine an example of exon skipping, we analyzed the Group B Cd44 gene, which contains a set of wellcharacterized alternative exons that are organized in tandem (exons 6 through 15) (Fig. 6A; Lynch 2004). These exons are excluded from the mature mRNA in macrophages (Fig. $6 \mathrm{~A}$ and data not shown). We find that spliced reads across the exon 4-exon 5 junction peak at $60 \mathrm{~min}, \sim 30 \mathrm{~min}$ after transcription through the intron (Fig. 6B, cf. red and black). In contrast, accumulation of spliced reads across the exon 5-exon 16 junction was delayed relative to all other junctions analyzed (Fig. 6C, black), including the $3^{\prime}$ terminal junction between exons 18 and 19 (Fig. 6D, black). These data confirm the interpretation of steady-state read enrichment within alternatively spliced introns, and indicate that skipping of regulated exons can cause a significant delay in splicing (Pandya-Jones and Black 2009; Khodor et al. 2011, 2012).

CD44 is a membrane protein, whose mRNA is associated with the ER. We previously showed that these mRNAs are enriched in the nucleoplasmic fraction, presumably due to the co-fractionation of endoplasmic reticulum (ER) with the contiguous nuclear envelope (Bhatt et al. 2012). This produces a background of Cd44 mRNA in the nucleoplasmic fraction even prior to induction. Nevertheless, we observe the increase in nucleoplasmic Cd44 mRNA after gene induction. Notably, this nucleoplasmic accumulation coincides with splicing across exons 5-16 (Fig. 6C), consistent with the idea that the intron with the slowest splicing kinetics within a transcript will determine the timing of release into the soluble nucleoplasm.

To look at other regions encompassing regulated exons, we examined the constitutively expressed gene $C d 45$. The Cd45 mRNA also contains a group of well-characterized alternative exons that appear to be predominantly skipped in macrophages (Fig. 7A; Tong et al. 2005). As this gene is equally transcribed at all time points, we cannot follow its synthesis and splicing over time. However, as observed on Cd44, there is a higher read density in the long Cd45 intron containing the skipped exons 4, 5, and 6 than elsewhere along the locus (Fig. 7A,B). This intron accumulation might, in part, result from Pol II pausing as described in a T-lymphocyte cell line (Shukla et al. 2011). However, reads for the exons downstream from exon 7 are enriched in the chromatin fraction indicating that they may be spliced prior to exons $3-$ 7 (Fig. 7A). Moreover, the transcriptional pausing in T-cells was associated with exon inclusion and not skipping, and in published data sets for LPS-stimulated macrophages there 


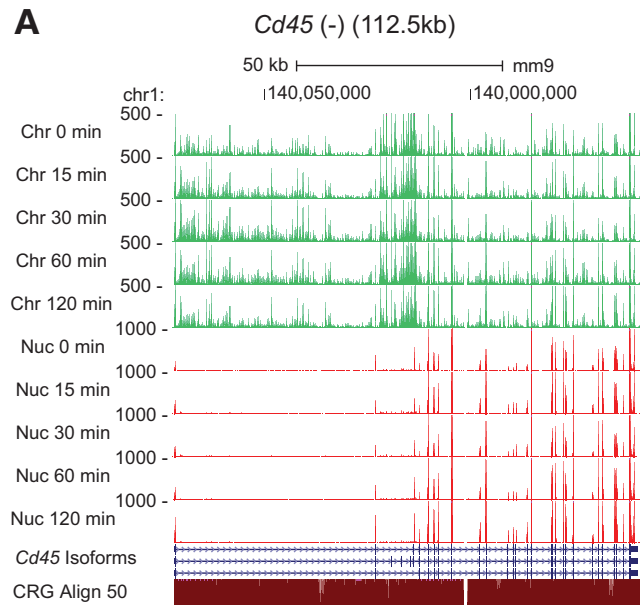

C Cd45 alternative region

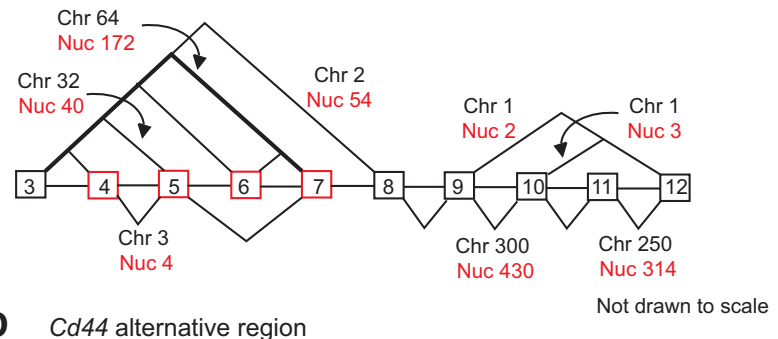

D Cd44 alternative region

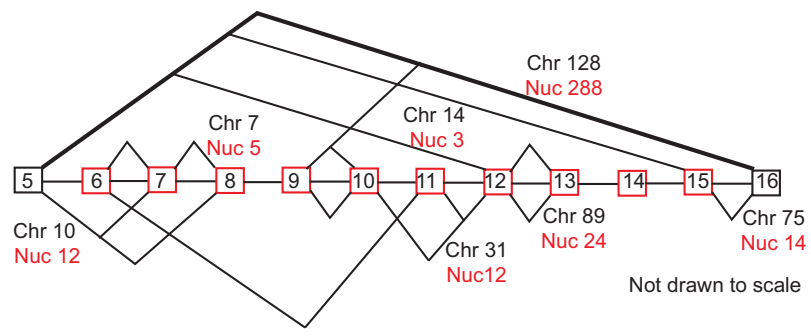

$\mathbf{E}$

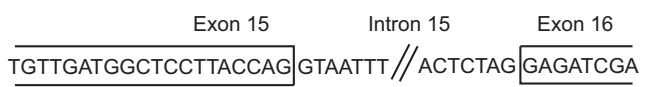

FIGURE 7. Aberrant splicing of skipped alternative exons. (A) Genome browser tracks of reads mapping to the 112.5 -kb $C d 45$ gene in the chromatin (green) and nucleoplasmic (red) fractions. This gene is constitutively expressed, thus each time point can be considered a biological replicate. (B) Line graph showing the total RPKM for each intron of the $C d 45$ gene in the chromatin and nucleoplasmic fractions, averaged over all five time points. Error bars represent the standard error of the mean. (C) Diagram of Cd45 exons 3-12. Exons 4-7 outlined in red are alternative, although in macrophages the dominant isoform includes exon 7. Diagonal lines join exons where chromatin-associated splicing events were observed. The predominant alternative splicing pattern is shown in bold. Numbers indicate the number of reads mapping to the indicated junction averaged over all five time points in the chromatin fraction. For clarity, the information for all junctions within the depicted region is not included. $(D)$ A diagram of the $C d 44$ alternative region. Constitutive exons 5 and 16 are outlined in black, and alternative exons 6-15 in red. Diagonal lines join exons where splicing events were observed. Bold lines depict productive splicing between exons 5 and 16. Numbers report the reads per event at 60 min on the chromatin. For clarity, the information for all junctions is not included. The values shown are representative of the range in read number per junction found across the $C d 44$ alternative region. (E) Sequence of the $3^{\prime}$ end of exon 15 and the $5^{\prime}$ end of exon 16 in the Cd44 gene.

is no obvious enrichment of Pol II ChIP-seq reads in this region (Escoubet-Lozach et al. 2011; Shukla et al. 2011). In macrophages, the increased chromatin-associated read density extends through the entire long intron encompassing all the skipped exons (Fig. 7A and B). These results are consistent with the observations of $\mathrm{Cd} 44$ that the splicing machinery takes longer to catalyze excision of long introns encompassing exons that will be excluded from the mature RNA.

\section{Splicing to cryptic splice sites and the incorrect joining of exons is rare}

Given the observed delays between the synthesis and splicing of some introns, we examined whether aberrant splicing could be observed in these regions. Within Cd44 intron 1, we found multiple instances of spliced reads that map to pairs of cryptic splice sites internal to the two correct sites (Supplemental Fig. S5). These reads were rare; only one or two reads were found for each aberrant event over all five time points, compared with hundreds of reads mapping to the correct junctions (Supplemental Fig. S5 and data not shown). Almost all the observed aberrant splicing events utilized GU-AG splice sites (Supplemental Fig. S5 and data not shown), indicating their likely origin from cryptic splicing by the spliceosome and not some other process. These cryptic splicing events in intron 1 are notable in that they will be eliminated from the final mRNA by the correct splicing of the intron (Supplemental Fig. S5).

Interestingly, we did not find a single read derived from the incorrect joining of two constitutive exons within $C d 44$ (data not shown). We did find some reads of this type in the $C d 45$ gene, albeit at extremely low frequency (Fig. 7C). The skipping of constitutive exons can generate mRNAs subject to nonsense-mediated decay (NMD). NMD requires translation and should lead to the loss of cytoplasmic mRNA and not the nuclear species. These observations suggest that the fidelity of splice site recognition by the spliceosome is high, or that processes that remove aberrantly spliced transcripts prior to template release are very efficient. 
Nevertheless, there is a measurable error rate, with some of the observed errors possibly eliminated by subsequent correct splicing.

Although the incorrect joining of constitutive exons appears to be rare, we found more significant numbers of reads derived from splicing of the variable exons in $C d 44$ and $C d 45$ (Fig. 7C,D; variable exons are highlighted in red). Both $C d 44$ and $C d 45$ encode membrane proteins whose final mRNAs fractionate with the nucleoplasm, preventing measurement of relative exon levels in the cytoplasm. In the nucleoplasm there are very few exon body reads for the variable exons of $C d 44$ and $C d 45$ (Figs. 6A, 7A). RPKM and total read numbers are not directly comparable between fractions because they are sampling from RNA populations with different overall complexities. As a general comparison, reads for correct exonexon junctions were generated in higher numbers from nucleoplasmic RNA than from chromatin, because the loss of intron sequence makes the spliced RNA a larger fraction of the nucleoplasmic RNA pool (data not shown). In contrast, reads for exon-exon joints involving the skipped exons decreased in nucleoplasm relative to chromatin, indicating a loss of the incorrectly spliced RNA (data not shown). On the chromatin, we find that spliced reads derived from the joining of skipped alternative exons occurred at higher frequencies than reads mapping to incorrectly paired constitutive exons. In fact, reads for certain alternative exon-exon junctions were nearly as abundant as those mapping to correctly joined constitutive exons (Fig. 7C,D). Thus, there is apparently a higher error rate for the joining of these alternative exons, even though these aberrant products are subsequently eliminated.

In $C d 44$, the variable exons are nested between exons 5 and 16 , where their exclusion from the mRNA would be achieved by excision of the long intron, whether they had previously been spliced to each other or not (Fig. 7D). Many of the most abundant splicing events involving the $C d 44$ variable exons could be eliminated in this way. In contrast, events joining a variable exon to one of the flanking constitutive exons could be eliminated by a second splicing event only if a new splice site was generated to allow subsequent splicing of exon 5 to exon 16. Interestingly, for exon 16, the only observed alternative splicing event involved splicing to exon 15 . This event was quite frequent, occurring on chromatin at $~ 60 \%$ the frequency of exon 5-16 splicing. Notably, the junction between exons 15 and 16 has potential to regenerate a $3^{\prime}$ splice site that could be used in resplicing of exon 16 to exon 5 , thus resolving the incorrect splicing event (Fig. 7E). In contrast, splicing events between exon 5 and the downstream alternative exons 7 and 8, and between $C d 45$ exons 3 and 5, would not generate new splice sites at the exon-exon junctions. These products would have to be eliminated by other mechanisms, perhaps through decay processes acting on incompletely spliced RNA. These findings indicate that some nonproductive splicing occurs between exons, and that this may be subsequently rescued by resplicing.

\section{DISCUSSION}

In this study, we characterize the relative kinetics of transcription, splicing, and transcript release from the chromatin compartment for genes of the inflammatory response. Stimulating primary macrophages with Lipid A, we induced transcription of a large set of endogenous genes. We applied RNA-seq to isolates of chromatin-associated and soluble nuclear RNA collected over time to measure the rates of exon ligation and transcript release from chromatin relative to RNA synthesis. This experimental approach allowed us to follow the RNA products as they were transcriptionally elongated, terminated, spliced, and then released into the nucleoplasm. We find that the rates of later steps in mRNA maturation strongly affect the timing of when a final protein product can be made after stimulation. This timing has important implications for understanding the inflammatory gene cascade. For example, Nfkbid, which is rapidly induced by Lipid A, encodes a nuclear inhibitor of NFkB. Unlike another immediate early gene, $c$ Fos, Nfkbid mRNA maturation and release are delayed by $\sim 15 \mathrm{~min}$, thereby slowing the negative feedback on NFkB activity that it controls. Thus, these rates of mRNA maturation and release will need to be incorporated into predictive models of the inflammatory gene response (Hetru and Hoffmann 2009).

We find that the appearance of some spliced exon-exon junctions can be substantially delayed relative to synthesis of the exons themselves and that introns in the Lipid A-induced gene set often are excised after the time needed to fully transcribe a locus. These introns can be substantially slower in their excision than other introns whose removal occurs prior to transcript completion. In contrast, $3^{\prime}$ end cleavage of nascent transcripts occurs rapidly after transcription across the poly(A) site. These differing kinetics result in pools of transcripts associated with chromatin that are cleaved and polyadenylated at the $3^{\prime}$ end but are not yet completely spliced (Fig. 8 ). This is consistent with previous results demonstrating that cleavage and polyadenylation can occur prior to splicing in adenoviral and mammalian transcripts, as well as with observations that pools of polyadenylated RNA can be observed at active gene loci (Nevins and Darnell 1978; Harpold et al. 1979; Weber et al. 1980; Kessler et al. 1993; Rigo and Martinson 2009; Brody et al. 2011).

We observe that a lag occurs after the completion of transcription before transcript release from the chromatin fraction into the nucleoplasm, and that RNA passes through this nucleoplasmic fraction prior to appearance in the cytoplasm (Fig. 8; Bhatt et al. 2012). Introns in sporadic constitutive genes are detectable in the nucleoplasmic fraction, and imaging data indicate that some introns may be excised away from their chromatin template (Supplemental Fig. S1; Vargas et al. 2011; Girard et al. 2012). However, measurements of steady-state RNA levels from most constitutive genes found intronic RNA sequence primarily in the chromatin fraction (Supplemental Fig. S1; Wuarin and Schibler 1994; 


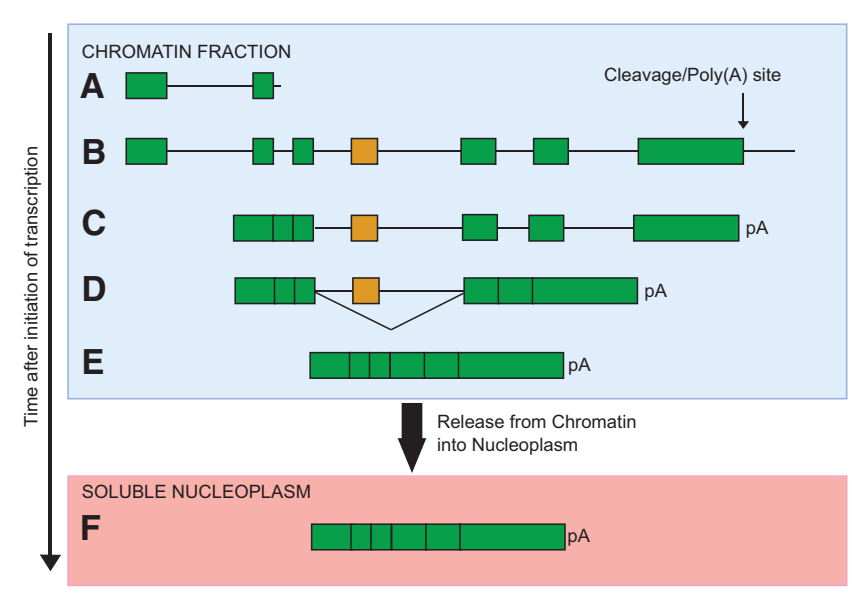

FIGURE 8. The sequence of maturation events observed for Lipid Ainduced transcripts. We find that splicing and transcript release from chromatin are slow relative to RNA synthesis and $3^{\prime}$ end cleavage. This gives rise to the following sequence of events for Lipid A-induced transcripts. (A) Transcription initiates upon Lipid A stimulation. (B) Transcription of the gene completes. Some splicing likely initiates prior to this, but for the genes assayed here, spliced read accumulation largely coincided with the time of transcript completion or later. $(C)$ Cleavage and polyadenylation at the $3^{\prime}$ splice site is rapid once transcription proceeds across the site. Introns are removed roughly in a $5^{\prime}$-to- $3^{\prime}$ order along the transcript. $(D)$ Introns encompassing an alternative exon that is to be excluded from the final message are spliced especially slowly. (E) Fully processed, mature mRNAs are released from the DNA template. $(F)$ RNA released into the nucleoplasm is largely complete.

Pandya-Jones and Black 2009; Khodor et al. 2011, 2012; Tilgner et al. 2012; and data not shown). If these transcripts become dissociated from the locus after transcription, they must rapidly rebind to components of the chromatin pellet. We find that serine/arginine (SR) proteins and other speckle components do not pellet with the chromatin and that the association of RNA with this fraction is sensitive to DNase treatment, indicating that its interaction requires intact DNA (data not shown). Thus, the RNA in the chromatin fraction is most likely still associated with the locus. However, this RNA is not necessarily still bound to the Pol II on the DNA template. The fraction presumably contains what have been called perispeckles that contain polyadenylated RNA and are adjacent to speckles and gene loci (Brody et al. 2011; Daguenet et al. 2012). Once released into the soluble nucleoplasm, transcripts rapidly appear in the cytoplasmic fraction, in agreement with relatively rapid rates of RNA nuclear export observed by microscopy (Ben-Ari et al. 2010; Grunwald and Singer 2010; Bhatt et al. 2012).

In the Lipid A-induced genes, we find nearly all introncontaining RNA within the chromatin fraction, and that RNA within the soluble nucleoplasm is fully spliced. For these Lipid A-induced genes, the release of RNA into the nucleoplasm coincided with the splicing of the slowest intron. Thus, the lag in transcript release from the chromatin may reflect the time needed to complete splicing. A requirement for complete splicing prior to release is consistent with many studies showing an accumulation of RNA at gene loci in response to perturbations of the splicing reaction or disruptions in mRNA export (Custodio et al. 1999; Jensen et al. 2001; Abruzzi et al. 2006; Brody et al. 2011; Martins et al. 2011). The coupling of final transcript maturation to template release would provide a site of action for quality control mechanisms that act on incorrectly processed RNAs prior to their entry into the nuclear export pathway. It will be very interesting to examine how factors involved in RNA quality control and export, such as the TREX/THO complexes, affect the distribution of mRNAs between the chromatin and nucleoplasmic fractions (Strasser et al. 2002; Rougemaille et al. 2008; Dias et al. 2010; Schmid and Jensen 2010).

Some introns are excised during RNA synthesis and others later (Bauren and Wieslander 1994; Tennyson et al. 1995; Wetterberg et al. 1996). Singh and Padgett (2009) made the most extensive measurements of splicing rates, examining constitutive transcripts restarted after DRB inhibition of transcription. They found that introns are typically excised $\sim 8$ min after synthesis of the $3^{\prime}$ exon. Although a large fraction of the introns we examined are excised at comparable rates, some are much slower. One interesting possibility is that the transcriptional induction process by Lipid A contributes to a delay in splicing for certain introns. Factors required for these slow introns may not be immediately recruited to a locus, and this would contribute to the timing of the evolving Lipid A response. In contrast, introns in transcripts from constitutively expressed genes may be tuned for rapid excision. However, the patterns of steady state RNA accumulation on the introns and exons of constitutive genes are highly variable, and this may be an indication of different rates of intron excision on these transcripts as well.

The source of the differing intron excision kinetics is not clear. In vitro, the rate of splicing can be altered by changing the strength of the $5^{\prime}$ splice site or the length of the intron, or by adding enhancer or silencer elements (Hertel and Maniatis 1998; Yu et al. 2008; Hicks et al. 2010). We did not observe correlations of either splice site strength or intron length with the rates of splicing (data not shown). The variability in intron excision rates presumably reflects rate differences in steps of spliceosome assembly and catalysis subsequent to initial splice site recognition and perhaps subsequent to splice site pairing.

Regulated introns can be particularly slow in their excision. In earlier observations, introns containing exons that will be excluded from the final mRNA were found to be present on the chromatin at higher levels than adjacent constitutive introns (Pandya-Jones and Black 2009; Khodor et al. 2011, 2012). In now measuring the excision rate of such an intron in $C d 44$, we find that it is indeed excised much more slowly than the other introns in the transcript. Splicing of complex regions that offer competing choices for spliceosome assembly may involve additional rate limiting regulatory steps prior to proper exon ligation. For example, there is evidence that SR proteins bound within introns are inhibitory for splicing 
(Kanopka et al. 1996; Ibrahim et al. 2005). Since exons that are excluded during splicing will likely bind SR proteins at their exonic splicing enhancer elements, these proteins must somehow be removed or ignored during splicing. These results underscore how little we know regarding the kinetics of spliceosome assembly, which steps are rate limiting, and how features of the pre-mRNA might alter those rates.

There is substantial evidence for cross-talk between transcription and splicing. Specifically, transcription elongation rates are thought to affect the choice of some alternative splicing patterns (Perales and Bentley 2009; de la Mata et al. 2010; Pandya-Jones 2011). Although intron excision can be slow relative to RNA synthesis, as we show here, splice site pairing choices may still occur during transcription. In fact, introns near the $5^{\prime}$ end of long genes do appear to be excised prior to introns near the $3^{\prime}$ end, even though some splicing catalysis is occurring long after the intron is synthesized; this may be indicative of the sequence of spliceosome assembly during transcription (de la Mata et al. 2010). On the other hand, the long delay in the excision of regulated introns relative to their synthesis offers additional opportunities for altering the choice of splicing patterns. There is evidence that regulatory proteins can alter splice site choice after the initial pairing of splice sites (Lim and Hertel 2004; Kotlajich et al. 2009). It will be interesting to examine whether pools of completed but unspliced transcripts can be differentially spliced depending on cellular conditions. It has been shown that a pool of partially spliced Clk1/4 mRNA is stored for later completion in response to stress, but this RNA does not appear to be at the Clk1/4 locus (Ninomiya et al. 2011).

In a previous study, we examined the splicing of $c-S r c$ and Fibronectin transcripts in chromatin and nucleoplasmic fractions of tissue culture cells by RT-PCR (Pandya-Jones and Black 2009). As described above, the results of this study were largely consistent with our observations here, except that we found increasing levels of intron sequence at the $3^{\prime}$ ends of genes. We interpreted the $5^{\prime}$-to- $3^{\prime}$ gradient in intron abundance as reflecting the excision of introns as polymerase progresses through the locus. This may be correct for $c-S r c$, $F n$, and other constitutive genes. However, these measurements were of steady-state RNA levels where the rates of splicing and transcription could not be directly assessed. We find here that the patterns of intron and exon abundance of constitutive genes measured at steady state are highly variable and that it is difficult to draw general conclusions about the order of intron excision from these data. Moreover, we find that intron excision may still progress in a roughly $5^{\prime}$-to- $3^{\prime}$ order along a transcript even if splicing initiates after transcription has proceeded through most of the gene.

Other recent studies also applied RNA-seq analysis to chromatin-associated RNA of constitutive genes (Khodor et al. 2011, 2012; Tilgner et al. 2012). Khodor and colleagues examined Drosophila melanogaster and rat liver genes at steady state, with the added step of removing poly $(\mathrm{A})^{+} \mathrm{RNA}$ from the chromatin RNA pool to ensure only nascent chains were pre- sent. While the differences in the experimental systems prevent the exact comparison of their results with ours, the authors similarly conclude that splicing largely occurs in the chromatin fraction and that regulated introns are slow to splice. The authors also report higher levels of introns in nascent mammalian transcripts than in Drosophila, indicating that splicing may be slower relative to RNA synthesis for mammalian genes (Khodor et al. 2012). Another genomewide metagene analysis of RNA-seq data from K562 cells also found a modest cross-gene gradient of ligated exons (Tilgner et al. 2012). These authors found that long noncoding RNAs may also have relatively slowly spliced introns, although again the rates could not be determined from steady-state conditions.

In another study, RNA-seq was applied to total human brain RNA. Saw-tooth patterns of decreasing read density observed across introns were interpreted as indicating rapid intron excision as the polymerase passed through a $3^{\prime}$ splice site (Ameur et al. 2011). This analysis was conducted on total, steady-state RNA containing both fully spliced nuclear and cytoplasmic RNA, as well as immature RNA presumably associated with the chromatin. This precluded examination of exon abundance in the nascent RNA. In examining steadystate genes that are not affected by Lipid A stimulation, we also find examples of saw-tooth patterns of read density across introns (Supplemental Fig. S1); however, these patterns are not pervasive across all introns nor all genes (see also Khodor et al. 2012). Moreover, although there is some enrichment of exon reads surrounding these introns, we do not observe decreasing $5^{\prime}$-to- $3^{\prime}$ gradients of exon reads along the length of these genes that would indicate very rapid intron excision during transcription. It is important to point out that there is wide variation in the patterns of RNA-seq read accumulation between individual genes, particularly at steady state. Thus, we would not rule out the possible rapid excision of some introns, including in $c$-Src and $F n$ transcripts (although also see Khodor et al. 2012). The pattern of decreasing $5^{\prime}$-to- $3^{\prime}$ read densities within introns was not generally seen in the Lipid A-induced transcripts, but again it is possible that there are differences in intron excision rates between some constitutive genes and Lipid A inducible genes.

The error rate of the splicing process is difficult to estimate in vivo. In analyzing total RNA or mRNA, a variety of quality control mechanisms have likely degraded many of the nonfunctional mRNAs produced from aberrant splicing patterns. We examined whether we might observe higher levels of aberrant products in our nascent RNA fraction. In general, the fidelity of splice site selection appears to be high, even when splicing is relatively slow and abundant unspliced RNA accumulates at a gene locus (Fox-Walsh and Hertel 2009). Nevertheless, we do find low levels of aberrant splicing events occurring in the analyzed transcription units. The more commonly observed errors appear to be splicing events that will be resolved by subsequent splicing at the correct junctions. These include splicing at cryptic splice sites internal to the 
correct sites and splicing between alternative exons that will not affect the final mRNA after excision of the long intron that contains them. It also appears that $C d 44$ exon 16 may join to exon 15 only to subsequently resplice to exon 5 . The resplicing of exon 16 is conceptually similar to the recursive splicing that has been described in a long intron of the Drosophila Ubx locus (Hatton et al. 1998; Burnette et al. 2005). In that case, a $5^{\prime}$ splice site is repeatedly respliced to a series of $3^{\prime}$ splice sites along the long intron. We did not observe intermediate spliced products for this kind of recursive splicing in the genes analyzed, but it may occur on other mammalian genes (although see Shepard et al. 2009). Aberrant spliced products that can be resolved by subsequent resplicing may not have a strong selection against their occurrence. Further analyses of nascent RNA after induction at greater sequence depth should allow more accurate measurement of splicing error rates.

This experimental approach can be applied to characterizing splicing responses to myriad other stimuli and cellular conditions. The isolation of the chromatin-associated RNA pool offers more rapid measurement of RNA changes than the traditional analysis of cytoplasmic mRNA. The method can also be applied to other RNA processing events, such as the maturation of microRNAs. A majority of miRNA genes are housed within introns and their processing is thought to occur in association with chromatin (Kim and Kim 2007; Pawlicki and Steitz 2008). We identified a miRNA within the Lipid A-induced gene set, miR1938 encoded within intron 1 of the Ifrd 1 gene (Fig. 3). We did not observe obvious differences in the excision kinetics for this intron, nor did the intron show unusually high read numbers after excision, as might be expected if the lariat product were particularly stable (data not shown). Using this approach, it will be interesting to assess where and when miRNA maturation occurs relative to the processing of host mRNAs. In this way, we hope to obtain much needed information on the maturation rates of a variety of transcripts.

\section{MATERIALS AND METHODS}

\section{Cell culture}

Bone marrow derived macrophages were isolated from mixed-sex C57BL/6 mice and cultured and stimulated with Lipid A as previously described (Bhatt et al. 2012).

\section{RNA isolation}

Cells were collected at $0,15,30,60$, and 120 min post-stimulation. Chromatin-associated and nucleoplasmic RNA fractions were isolated and characterized as previously described (Pandya-Jones and Black 2009; Bhatt et al. 2012). To estimate the loss of RNA from the chromatin into the nucleoplasm during purification, we examined the nuclear Xist transcripts. We found that $>95 \%$ of the total Xist RNA reads at all time points were found in the chromatin fraction (Supplemental Fig. S1A), as would be expected given its func- tion in initiating $X$ chromosome silencing (Wutz 2011). Read numbers between the chromatin and nucleoplasmic fractions cannot be compared directly, because of differences in library construction and the sequence complexity between the two fractions. Nevertheless, the chromatin fraction generated 20-40 times more $X i s t$ reads than the nucleoplasm, indicating that the Xist association with chromatin was stable to the fractionation. These data indicate that there is little loss of RNA from the chromatin into the nucleoplasm during purification and that the two fractions are composed of distinct RNA populations.

\section{Library construction}

Chromatin-associated and nucleoplasmic RNA fractions were subjected to library preparation as outlined in the Illumina mRNA library preparation kit. Ribosomal RNA was removed using the Ribominus kit (Invitrogen). A modification to the protocol involved the incorporation of dUTP into the second strand synthesis as described in Levin et al. (2010) to create a strand-specific library.

\section{Mapping statistics}

Fifty-nucleotide reads were aligned to the mouse mm9 male canonical genome using TopHat on Galaxy (options: $-\mathrm{a}=6 ;-\mathrm{m}=0 ;-\mathrm{i}$ $=50 ;-\mathrm{I}=500,000 ;-\mathrm{F}=0 ;-\mathrm{g}=1$; segment length $=25$, segment mismatch $=2$ ) (Giardine et al. 2005; Trapnell et al. 2009; Blankenberg et al. 2010; Goecks et al. 2010).

\section{Normalization and RPKM calculations}

Reads were assigned to genic regions using the RefSeq gene database and the BEDTools suite (Quinlan and Hall 2010). Exon and intron body reads were calculated using the annotated UCSC known gene database and the BEDTools commands coverageBed piped after intersectBed with flag $-\mathrm{f}$ 1.0. The same command was used to extract the $3^{\prime}$ intron junction reads, except $-\mathrm{f} 0.02$, since we required that the reads overlap the splice site by $1 \mathrm{nt}$. The spliced exon-exon reads were identified using the intersectBed command and curated manually. The RPKM values were calculated by normalizing the number of reads mapping to a feature by the total number of reads mapped per sample, as well as the length of the feature in kilobase. For spliced reads crossing an exon-exon junction, the feature size was set at $88 \mathrm{nt}$ because we required a 6-nt overlap across the junction. We limited analysis of ligated exon junctions to those with $\geq 15$ mapped reads at peak expression, with the vast majority of junctions accumulating between 25 and 200 reads at this time point (Pan et al. 2008). For the $3^{\prime}$ intron junction reads, feature length was set at $98 \mathrm{nt}$ to force a 1-nt overlap with the $3^{\prime}$ splice site.

\section{Quantitative RT-PCR}

Five-hundred nanograms of input RNA, as quantified on a nanodrop-1000 spectrophotometer, was random-primed according to the manufacturers protocol (Superscript III, Invitrogen). One-tenth of the cDNA and appropriate primers were added to LightCycler qPCR mix (Roche) and used in a qPCR reaction as described by the manufacturer. All qPCR reactions were performed on an MX300P machine (Stratagene). The efficiency of each primer set was deter- 
mined using a standard curve and quantification was normalized against absolute values of U6 snRNA. Primer sequences used in the analysis of Nfkbid are as follows:

Reverse primer against uncleaved RNA: ccccactccccatttttaat Reverse primer against poly(A) RNA: ttttttttttttttttttttttctaa Exon 11 forward: GGAGTTTCCATGGGATTTGA Exon 11 reverse: CACAGACCAAGGGGAACAAT Exon 9 forward: CCATTCTGCACTTGGCTGTA Intron 9 forward: ggttcaaagacccagagacg Exon 10 reverse: GCAGTGTCGGGTCTGCTC

\section{DATA DEPOSITION}

Sequencing data have been submitted to GEO under accession number GSE32916.

\section{SUPPLEMENTAL MATERIAL}

Supplemental material is available for this article.

\section{ACKNOWLEDGMENTS}

We thank Jim Darnell, Phil Sharp, Tim Nilsen, and members of the Black and Smale labs for helpful discussions and/or for advice on the manuscript. This work was supported by a UCLA Dissertation Year Fellowship (to A.P-J.) and by NIH grants R01GM086372 and R01CA127279 (to S.T.S.), and by the Broad Stem Cell Research Center at UCLA. D.L.B. is an Investigator of the Howard Hughes Medical Institute.

Received February 27, 2013; accepted March 13, 2013.

\section{REFERENCES}

Abruzzi KC, Belostotsky DA, Chekanova JA, Dower K, Rosbash M. 2006. 3 '-end formation signals modulate the association of genes with the nuclear periphery as well as mRNP dot formation. EMBO J 25: 4253-4262.

Alexander RD, Barrass JD, Dichtl B, Kos M, Obtulowicz T, Robert MC, Koper M, Karkusiewicz I, Mariconti L, Tollervey D, et al. 2010a. RiboSys, a high-resolution, quantitative approach to measure the in vivo kinetics of pre-mRNA splicing and $3^{\prime}$-end processing in Saccharomyces cerevisiae. RNA 16: 2570-2580.

Alexander RD, Innocente SA, Barrass JD, Beggs JD. 2010b. Splicing-dependent RNA polymerase pausing in yeast. Mol Cell 40: 582-593.

Ameur A, Zaghlool A, Halvardson J, Wetterbom A, Gyllensten U, Cavelier L, Feuk L. 2011. Total RNA sequencing reveals nascent transcription and widespread co-transcriptional splicing in the human brain. Nat Struct Mol Biol 18: 1435-1440.

Ardehali MB, Lis JT. 2009. Tracking rates of transcription and splicing in vivo. Nat Struct Mol Biol 16: 1123-1124.

Bauren G, Wieslander L. 1994. Splicing of Balbiani ring 1 gene premRNA occurs simultaneously with transcription. Cell 76: 183-192.

Ben-Ari Y, Brody Y, Kinor N, Mor A, Tsukamoto T, Spector DL, Singer RH, Shav-Tal Y. 2010. The life of an mRNA in space and time. J Cell Sci 123: 1761-1774.

Bhatt DM, Pandya-Jones A, Tong AJ, Barozzi I, Lissner MM, Natoli G, Black DL, Smale ST. 2012. Transcript dynamics of proinflammatory genes revealed by sequence analysis of subcellular RNA fractions. Cell 150: 279-290.
Bird G, Fong N, Gatlin JC, Farabaugh S, Bentley DL. 2005. Ribozyme cleavage reveals connections between mRNA release from the site of transcription and pre-mRNA processing. Mol Cell 20: 747-758.

Blankenberg D, Von Kuster G, Coraor N, Ananda G, Lazarus R, Mangan M, Nekrutenko A, Taylor J. 2010. Galaxy: A web-based genome analysis tool for experimentalists. Curr Protoc Mol Biol Chapter 19: Unit 1910 11-21.

Brody Y, Neufeld N, Bieberstein N, Causse SZ, Bohnlein EM, Neugebauer KM, Darzacq X, Shav-Tal Y. 2011. The in vivo kinetics of RNA polymerase II elongation during co-transcriptional splicing. PLoS Biol 9: e1000573.

Burnette JM, Miyamoto-Sato E, Schaub MA, Conklin J, Lopez AJ. 2005. Subdivision of large introns in Drosophila by recursive splicing at nonexonic elements. Genetics 170: 661-674.

Carrillo Oesterreich F, Preibisch S, Neugebauer KM. 2010. Global analysis of nascent RNA reveals transcriptional pausing in terminal exons. Mol Cell 40: 571-581.

Chan S, Choi EA, Shi Y. 2011. Pre-mRNA 3'-end processing complex assembly and function. Wiley Interdiscip Rev RNA 2: 321-335.

Cho EJ, Takagi T, Moore CR, Buratowski S. 1997. mRNA capping enzyme is recruited to the transcription complex by phosphorylation of the RNA polymerase II carboxy-terminal domain. Genes Dev 11: 3319-3326.

Core LJ, Waterfall JJ, Lis JT. 2008. Nascent RNA sequencing reveals widespread pausing and divergent initiation at human promoters. Science 322: 1845-1848.

Custodio N, Carmo-Fonseca M, Geraghty F, Pereira HS, Grosveld F, Antoniou M. 1999. Inefficient processing impairs release of RNA from the site of transcription. EMBO J 18: 2855-2866.

Daguenet E, Baguet A, Degot S, Schmidt U, Alpy F, Wendling C, Spiegelhalter C, Kessler P, Rio MC, Le Hir H, et al. 2012. Perispeckles are major assembly sites for the exon junction core complex. Mol Biol Cell 23: 1765-1782.

Darnell JE Jr. 2013. Reflections on the history of pre-mRNA processing and highlights of current knowledge: A unified picture. RNA 19: 443-460.

Darzacq X, Shav-Tal Y, de Turris V, Brody Y, Shenoy SM, Phair RD, Singer RH. 2007. In vivo dynamics of RNA polymerase II transcription. Nat Struct Mol Biol 14: 796-806.

de la Mata M, Lafaille C, Kornblihtt AR. 2010. First come, first served revisited: Factors affecting the same alternative splicing event have different effects on the relative rates of intron removal. RNA 16: 904-912.

De Santa F, Narang V, Yap ZH, Tusi BK, Burgold T, Austenaa L, Bucci G, Caganova M, Notarbartolo S, Casola S, et al. 2009. Jmjd3 contributes to the control of gene expression in LPS-activated macrophages. EMBO J 28: 3341-3352.

Derman E, Darnell JE. 1974. Relationship of chain transcription to poly (A) addition and processing of hnRNA in HeLa cells. Cell 3: 255-264.

Dias AP, Dufu K, Lei H, Reed R. 2010. A role for TREX components in the release of spliced mRNA from nuclear speckle domains. Nat Commun 1: 97.

Escoubet-Lozach L, Benner C, Kaikkonen MU, Lozach J, Heinz S, Spann NJ, Crotti A, Stender J, Ghisletti S, Reichart D, et al. 2011. Mechanisms establishing TLR4-responsive activation states of inflammatory response genes. PLoS Genet 7: e1002401.

Fox-Walsh KL, Hertel KJ. 2009. Splice-site pairing is an intrinsically high fidelity process. Proc Natl Acad Sci 106: 1766-1771.

Giardine B, Riemer C, Hardison RC, Burhans R, Elnitski L, Shah P, Zhang Y, Blankenberg D, Albert I, Taylor J, et al. 2005. Galaxy: A platform for interactive large-scale genome analysis. Genome Res 15: 1451-1455.

Girard C, Will CL, Peng J, Makarov EM, Kastner B, Lemm I, Urlaub H, Hartmuth K, Luhrmann R. 2012. Post-transcriptional spliceosomes are retained in nuclear speckles until splicing completion. Nat Commun 3: 994.

Goecks J, Nekrutenko A, Taylor J. 2010. Galaxy: A comprehensive approach for supporting accessible, reproducible, and transparent computational research in the life sciences. Genome Biol 11: R86. 
Grunwald D, Singer RH. 2010. In vivo imaging of labelled endogenous $\beta$-actin mRNA during nucleocytoplasmic transport. Nature 467: 604-607.

Harpold MM, Dobner PR, Evans R, Bancroft FC, Darnell JE Jr. 1979. The synthesis and processing of a nuclear RNA precursor to rat pregrowth hormone messenger RNA. Nucleic Acids Res 6: 31333144.

Hatton AR, Subramaniam V, Lopez AJ. 1998. Generation of alternative Ultrabithorax isoforms and stepwise removal of a large intron by resplicing at exon-exon junctions. Mol Cell 2: 787-796.

Hertel KJ, Maniatis T. 1998. The function of multisite splicing enhancers. Mol Cell 1: 449-455.

Hetru C, Hoffmann JA. 2009. NF-kB in the immune response of Drosophila. Cold Spring Harb Perspect Biol 1: a000232.

Hicks MJ, Mueller WF, Shepard PJ, Hertel KJ. 2010. Competing upstream $5^{\prime}$ splice sites enhance the rate of proximal splicing. Mol Cell Biol 30: 1878-1886.

Hocine S, Singer RH, Grunwald D. 2010. RNA processing and export. Cold Spring Harb Perspect Biol 2: a000752.

Hsin JP, Manley JL. 2012. The RNA polymerase II CTD coordinates transcription and RNA processing. Genes Dev 26: 2119-2137.

Ibrahim EC, Schaal TD, Hertel KJ, Reed R, Maniatis T. 2005. Serine/arginine-rich protein-dependent suppression of exon skipping by exonic splicing enhancers. Proc Natl Acad Sci 102: 5002-5007.

Jensen TH, Patricio K, McCarthy T, Rosbash M. 2001. A block to mRNA nuclear export in S. cerevisiae leads to hyperadenylation of transcripts that accumulate at the site of transcription. Mol Cell 7: 887-898.

Kanopka A, Muhlemann O, Akusjarvi G. 1996. Inhibition by SR proteins of splicing of a regulated adenovirus pre-mRNA. Nature 381: 535-538.

Kessler O, Jiang Y, Chasin LA. 1993. Order of intron removal during splicing of endogenous adenine phosphoribosyltransferase and dihydrofolate reductase pre-mRNA. Mol Cell Biol 13: 6211-6222.

Khodor YL, Rodriguez J, Abruzzi KC, Tang CH, Marr MT II, Rosbash M. 2011. Nascent-seq indicates widespread cotranscriptional pre-mRNA splicing in Drosophila. Genes Dev 25: 2502 2512.

Khodor YL, Menet JS, Tolan M, Rosbash M. 2012. Cotranscriptional splicing efficiency differs dramatically between Drosophila and mouse. RNA 18: 2174-2186.

Kim YK, Kim VN. 2007. Processing of intronic microRNAs. EMBO J 26: 775-783.

Kotlajich MV, Crabb TL, Hertel KJ. 2009. Spliceosome assembly pathways for different types of alternative splicing converge during commitment to splice site pairing in the A complex. Mol Cell Biol 29: $1072-1082$.

Lacadie SA, Tardiff DF, Kadener S, Rosbash M. 2006. In vivo commitment to yeast cotranscriptional splicing is sensitive to transcription elongation mutants. Genes Dev 20: 2055-2066.

LeMaire MF, Thummel CS. 1990. Splicing precedes polyadenylation during Drosophila E74A transcription. Mol Cell Biol 10: 6059-6063.

Levin JZ, Yassour M, Adiconis X, Nusbaum C, Thompson DA, Friedman N, Gnirke A, Regev A. 2010. Comprehensive comparative analysis of strand-specific RNA sequencing methods. Nat Methods 7: 709-715.

Lim SR, Hertel KJ. 2004. Commitment to splice site pairing coincides with A complex formation. Mol Cell 15: 477-483.

Listerman I, Sapra AK, Neugebauer KM. 2006. Cotranscriptional coupling of splicing factor recruitment and precursor messenger RNA splicing in mammalian cells. Nat Struct Mol Biol 13: 815-822.

Lynch KW. 2004. Consequences of regulated pre-mRNA splicing in the immune system. Nat Rev Immunol 4: 931-940.

Martins SB, Rino J, Carvalho T, Carvalho C, Yoshida M, Klose JM, de Almeida SF, Carmo-Fonseca M. 2011. Spliceosome assembly is coupled to RNA polymerase II dynamics at the $3^{\prime}$ end of human genes. Nat Struct Mol Biol 18: 1115-1123.

Matlin AJ, Moore MJ. 2007. Spliceosome assembly and composition. Adv Exp Med Biol 623: 14-35.
McCracken S, Fong N, Rosonina E, Yankulov K, Brothers G, Siderovski D, Hessel A, Foster S, Shuman S, Bentley DL. 1997. 5'Capping enzymes are targeted to pre-mRNA by binding to the phosphorylated carboxy-terminal domain of RNA polymerase II. Genes Dev 11: 3306-3318.

Nevins JR, Darnell JE Jr. 1978. Steps in the processing of Ad2 mRNA: Poly $(\mathrm{A})^{+}$nuclear sequences are conserved and poly(A) addition precedes splicing. Cell 15: 1477-1493.

Ninomiya K, Kataoka N, Hagiwara M. 2011. Stress-responsive maturation of Clk1/4 pre-mRNAs promotes phosphorylation of SR splicing factor. J Cell Biol 195: 27-40.

Osheim YN, Miller OL Jr, Beyer AL. 1985. RNP particles at splice junction sequences on Drosophila chorion transcripts. Cell 43: 143-151.

Pan Q, Shai O, Lee LJ, Frey BJ, Blencowe BJ. 2008. Deep surveying of alternative splicing complexity in the human transcriptome by high-throughput sequencing. Nat Genet 40: 1413-1415.

Pandya-Jones A. 2011. Pre-mRNA splicing during transcription in the mammalian system. Wiley Interdiscip Rev RNA 2: 700-717.

Pandya-Jones A, Black DL. 2009. Co-transcriptional splicing of constitutive and alternative exons. RNA 15: 1896-1908.

Pawlicki JM, Steitz JA. 2008. Primary microRNA transcript retention at sites of transcription leads to enhanced microRNA production. J Cell Biol 182: 61-76.

Perales R, Bentley D. 2009. "Cotranscriptionality": The transcription elongation complex as a nexus for nuclear transactions. Mol Cell 36: $178-191$.

Quinlan AR, Hall IM. 2010. BEDTools: A flexible suite of utilities for comparing genomic features. Bioinformatics 26: 841-842.

Rabani M, Levin JZ, Fan L, Adiconis X, Raychowdhury R, Garber M, Gnirke A, Nusbaum C, Hacohen N, Friedman N, et al. 2011. Metabolic labeling of RNA uncovers principles of RNA production and degradation dynamics in mammalian cells. Nat Biotechnol 29: 436-442.

Ramirez-Carrozzi VR, Braas D, Bhatt DM, Cheng CS, Hong C, Doty KR, Black JC, Hoffmann A, Carey M, Smale ST. 2009. A unifying model for the selective regulation of inducible transcription by $\mathrm{CpG}$ islands and nucleosome remodeling. Cell 138: 114-128.

Rigo F, Martinson HG. 2009. Polyadenylation releases mRNA from RNA polymerase II in a process that is licensed by splicing. RNA 15: $823-836$.

Rougemaille M, Dieppois G, Kisseleva-Romanova E, Gudipati RK, Lemoine S, Blugeon C, Boulay J, Jensen TH, Stutz F, Devaux F, et al. 2008. THO/Sub2p functions to coordinate $3^{\prime}$-end processing with gene-nuclear pore association. Cell 135: 308-321.

Salditt-Georgieff M, Harpold M, Sawicki S, Nevins J, Darnell JE Jr. 1980. Addition of poly(A) to nuclear RNA occurs soon after RNA synthesis. J Cell Biol 86: 844-848.

Schmid M, Jensen TH. 2010. Nuclear quality control of RNA polymerase II transcripts. Wiley Interdiscip Rev RNA 1: 474-485.

Schmidt U, Basyuk E, Robert MC, Yoshida M, Villemin JP, Auboeuf D, Aitken S, Bertrand E. 2011. Real-time imaging of cotranscriptional splicing reveals a kinetic model that reduces noise: Implications for alternative splicing regulation. J Cell Biol 193: 819-829.

Selth LA, Sigurdsson S, Svejstrup JQ. 2010. Transcript elongation by RNA Polymerase II. Annu Rev Biochem 79: 271-293.

Shepard S, McCreary M, Fedorov A. 2009. The peculiarities of large intron splicing in animals. PLoS One 4: e7853.

Shukla S, Kavak E, Gregory M, Imashimizu M, Shutinoski B, Kashlev M, Oberdoerffer P, Sandberg R, Oberdoerffer S. 2011. CTCF-promoted RNA polymerase II pausing links DNA methylation to splicing. Nature 479: 74-79.

Shuman S. 2001. Structure, mechanism, and evolution of the mRNA capping apparatus. Prog Nucleic Acid Res Mol Biol 66: 1-40.

Singh J, Padgett RA. 2009. Rates of in situ transcription and splicing in large human genes. Nat Struct Mol Biol 16: 1128-1133.

Stewart M. 2010. Nuclear export of mRNA. Trends Biochem Sci 35: 609-617.

Strasser K, Masuda S, Mason P, Pfannstiel J, Oppizzi M, RodriguezNavarro S, Rondon AG, Aguilera A, Struhl K, Reed R, et al. 2002. 
TREX is a conserved complex coupling transcription with messenger RNA export. Nature 417: 304-308.

Tennyson CN, Klamut HJ, Worton RG. 1995. The human dystrophin gene requires 16 hours to be transcribed and is cotranscriptionally spliced. Nat Genet 9: 184-190.

Tilgner H, Knowles DG, Johnson R, Davis CA, Chakrabortty S, Djebali S, Curado J, Snyder M, Gingeras TR, Guigo R. 2012. Deep sequencing of subcellular RNA fractions shows splicing to be predominantly cotranscriptional in the human genome but inefficient for lncRNAs. Genome Res 22: 1616-1625.

Tong A, Nguyen J, Lynch KW. 2005. Differential expression of CD45 isoforms is controlled by the combined activity of basal and inducible splicing-regulatory elements in each of the variable exons. J Biol Chem 280: 38297-38304.

Trapnell C, Pachter L, Salzberg SL. 2009. TopHat: Discovering splice junctions with RNA-Seq. Bioinformatics 25: 1105-1111.

Vargas DY, Shah K, Batish M, Levandoski M, Sinha S, Marras SA, Schedl P, Tyagi S. 2011. Single-molecule imaging of transcriptionally coupled and uncoupled splicing. Cell 147: 1054-1065.
Wahl MC, Will CL, Luhrmann R. 2009. The spliceosome: Design principles of a dynamic RNP machine. Cell 136: 701-718.

Weber J, Blanchard JM, Ginsberg H, Darnell JE Jr. 1980. Order of polyadenylic acid addition and splicing events in early adenovirus mRNA formation. J Virol 33: 286-291.

Wetterberg I, Bauren G, Wieslander L. 1996. The intranuclear site of excision of each intron in Balbiani ring 3 pre-mRNA is influenced by the time remaining to transcription termination and different excision efficiencies for the various introns. RNA 2: 641-651.

Wuarin J, Schibler U. 1994. Physical isolation of nascent RNA chains transcribed by RNA polymerase II: Evidence for cotranscriptional splicing. Mol Cell Biol 14: 7219-7225.

Wutz A. 2011. Gene silencing in X-chromosome inactivation: Advances in understanding facultative heterochromatin formation. Nat Rev Genet 12: 542-553.

Yu Y, Maroney PA, Denker JA, Zhang XH, Dybkov O, Luhrmann R, Jankowsky E, Chasin LA, Nilsen TW. 2008. Dynamic regulation of alternative splicing by silencers that modulate $5^{\prime}$ splice site competition. Cell 135: 1224-1236. 

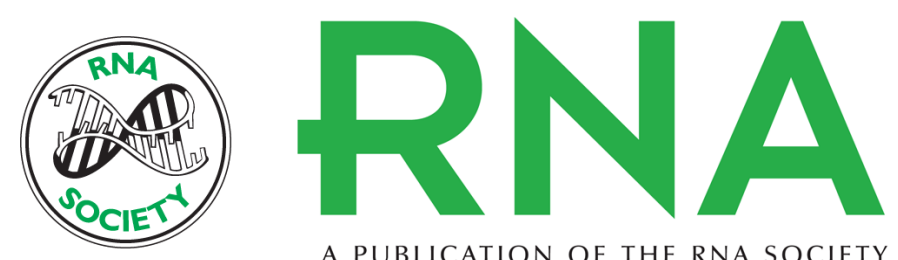

A PUBLICATION OF THE RNA SOCIETY

\section{Splicing kinetics and transcript release from the chromatin compartment limit the rate of Lipid A-induced gene expression}

Amy Pandya-Jones, Dev M. Bhatt, Chia-Ho Lin, et al.

RNA 2013 19: 811-827 originally published online April 24, 2013

Access the most recent version at doi:10.1261/rna.039081.113

\section{Supplemental http://rnajournal.cshlp.org/content/suppl/2013/04/08/rna.039081.113.DC1 \\ Material}

References This article cites 87 articles, 34 of which can be accessed free at: http://rnajournal.cshlp.org/content/19/6/811.full.html\#ref-list-1

Open Access Freely available online through the RNA Open Access option.

License Freely available online through the RNA Open Access option.

Email Alerting Receive free email alerts when new articles cite this article - sign up in the box at the Service top right corner of the article or click here.

\section{|||||||| Providing Precise Solutions for} your research.

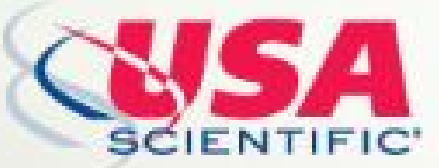

To subscribe to $R N A$ go to:

http://rnajournal.cshlp.org/subscriptions 